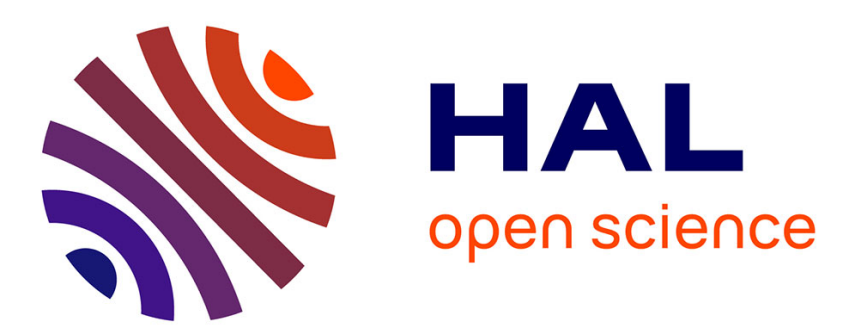

\title{
Some examples of friction-induced vibrations and instabilities
}

\author{
Franck Moirot, Quoc-Son Nguyen
}

\section{To cite this version:}

Franck Moirot, Quoc-Son Nguyen. Some examples of friction-induced vibrations and instabilities. J.A.C. Martins; M. Raous. Friction and Instabilities, Spring, pp.137-179, 2002, International Centre for Mechanical Sciences, 10.1007/978-3-7091-2534-2_4 . hal-00112295

\section{HAL Id: hal-00112295 \\ https://hal.science/hal-00112295}

Submitted on 31 Oct 2018

HAL is a multi-disciplinary open access archive for the deposit and dissemination of scientific research documents, whether they are published or not. The documents may come from teaching and research institutions in France or abroad, or from public or private research centers.
L'archive ouverte pluridisciplinaire HAL, est destinée au dépôt et à la diffusion de documents scientifiques de niveau recherche, publiés ou non, émanant des établissements d'enseignement et de recherche français ou étrangers, des laboratoires publics ou privés. 


\title{
Some examples of friction-induced vibrations and instabilities
}

\author{
Franck Moirot ${ }^{1}$ and Quoc-Son Nguyen ${ }^{2}$ \\ 1 PSA, Centre Technique, Paris \\ ${ }^{2}$ CNRS, Laboratoire de Mécanique des Solides, Ecole Polytechnique, Paris, France
}

\begin{abstract}
Some examples of friction-induced vibrations and instabilities of elastic structures in unilateral contact are discussed in this paper. The case of an elastic solid in contact with a moving (rigid or elastic) obstacle is considered. The onset of instability and the transition to a dynamic regime is the underlying centre of interest of the discussion.

Part 1 gives an overview of some classical and basic results concerning the stability analysis of an equilibrium. The linearization method and Liapunov's theorem are first recalled. The possibility of Hopf's bifurcation is considered when there is flutter instability. For timeindependent standard dissipative systems, the description of the rate problem and the criteria of static stability and rate uniqueness in the sense of Hill are discussed.

Part 2 deals with the instability of the steady sliding of an elastic structure in contact with friction with a rigid or elastic obstacle. Closed-form solutions are discussed for some simple systems and for the problem of frictional contact of elastic layers.

The possibility of stick-slip vibrations is discussed in Part 3 in an analytical example of two encased cylinders. The existence of a family of stick-slip waves propagating at constant velocity and with positive slip is discussed.

Part 4 addresses the problem of brake squeal as a direct application of the theoretical analysis. This phenomenon is interpreted here as a consequence of the flutter instability of the steady sliding solution. A numerical analysis by the finite element method is performed to compute the steady sliding solution and to discuss its stability for an automotive disk brake.
\end{abstract}

\section{Introduction and scope}

The example of a simple oscillator in frictional contact with a moving obstacle, as shown in Figure 1, is first considered in order to discuss its dynamic behaviour. It is assumed that the friction coefficient may depend on the sliding speed $f=f(v)$. The particular case $f(0)=f_{s}$ and $f(v)=f_{d}<f_{s}$ corresponds to the definition of a static coefficient $f_{s}$ and of a dynamic coefficient $f_{d}$. The equation of motion

$$
M \ddot{x}+K x=f M g \operatorname{sign}(w-\dot{x})
$$

then leads to the phase diagram shown in Figure 2 (upper graph). Starting from any initial condition, the motion of the mass will become periodic.

If the initial condition is very close to the equilibrium, the motion is the natural harmonic vibration of the mass within the slip regime. If the initial condition is far from the equilibrium, the limit dynamic response consists of two phases, a stick phase of duration $\Delta_{k}=\frac{2\left(f_{s}-f_{d}\right) M g}{K w}$ and a slip phase $\Delta_{p}=2 \pi-2 \arctan \left(\frac{\Delta_{k} \sqrt{K / M}}{2}\right)$. The frequency of the limit periodic motion 


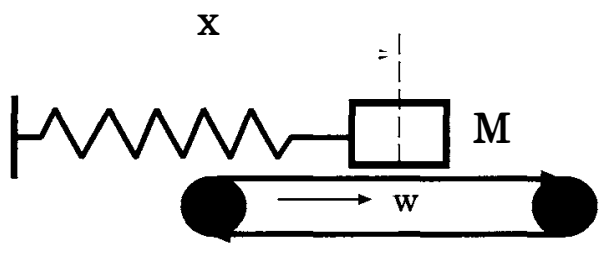

Figure 1: A simple frictional oscillator.

is lower than the natural vibration frequency $2 \pi \sqrt{\frac{M}{K}}$ of the simple oscillator. In particular, the stick phase disappears when $f_{s}=f_{d}$ i.e, when the friction cofficient is constant. The response is simply the slip oscillation of the oscillator with its natural frequency.

The results are quite similar for more complicated expressions of the friction coefficient in terms of the sliding speed. Figure 2 (lower graph) gives the results obtained with a linearly decreasing friction coefficient. In this case, the steady sliding response is unstable. A small perturbation of this solution leads to a stable limit cycle which is an attracting periodic solution as shown in this figure.

In this simple example, the stability of the steady sliding solution and the dynamic behaviour of the system associated with an arbitrary initial condition have been obtaind in a simple way. Our objective is to extend the discussion to more complicated discrete or continuous systems of solids.

The problem of vibrations and instabilities induced by friction is the centre of interest of this paper. This problem is considered for the system of an elastic solid in frictional contact with a moving obstacle which can be a rigid or elastic solid. The dynamic behaviour of the system is investigated. The stability of the steady sliding solution is discussed. The onset of instabilities and the subsequent dynamic response of the system after perturbations are the underlying subjects of interest. It is well known that the unilateral contact and Coulomb's law are principal sources of nonlinearity and that these nonlinearities cannot be discussed in the classical framework of stability and bifurcation analysis, for example by the classical proof of Hopf's bifurcation. However, partial answers to these difficult questions do exist and some interesting results have been recently obtained in the literature.

\section{Overview of stability}

\subsection{Stability of an equilibrium}

Let us consider a system in evolution defined by parameters $y(t)$ and governed by a differential equation

$$
\dot{y}=F(y)
$$



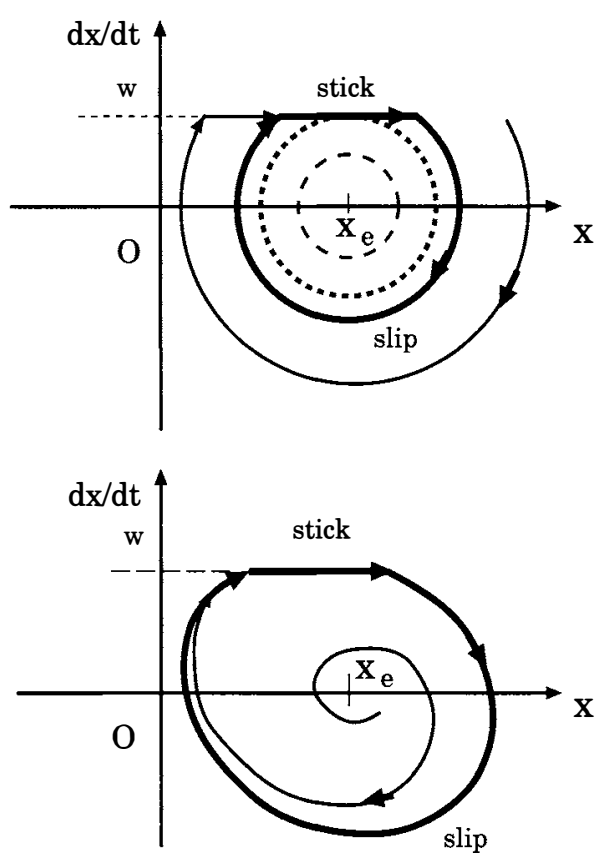

Figure 2: Stick-slip vibrations of a simple oscillator in contact with a moving obstacle. The upper diagram gives the motion in the phase plane when the static coefficient is greater than the dynamic friction coefficient. The lower diagram gives the obtained motion for a decreasing function $f(v)$, cf. Oestreich et al., 1996 and Moirot, 1998.

with initial condition $y(0)=y_{o}$. It is first assumed that $y$ is a vector in $\mathbf{R}^{n}$ and $F$ is a regular function of $y$ that does not depend on time $t$. Thus, only the case of autonomous equations is considered here.

A solution $y(t)$, associated with an initial condition $y(0)=y_{o}$, is by definition a dynamic evolution of the considered system. An equilibrium is a particular time-independent evolution i.e. $y(t)=y_{e}$ constant for all $t$. Thus, an equilibrium $y_{e}$ must necessarily satisfy

$$
F\left(y_{e}\right)=0
$$

The case of autonomous differential equations of higher-order also enters in this framework since they can be brought back to equations of order 1 by a change of variables.

By definition, an equilibrium position $y_{e}$ is stable if a small perturbation of this position will lead only to a small perturbed evolution of the system near the equilibrium. More precisely, if a measure $d(t)$ of the distance for time $t \geq 0$ of the perturbed evolution to the equilibrium can be introduced

$$
d(t)=\left\|y(t)-y_{e}\right\|
$$

the definition of stability can be written as

$$
y_{e} \text { stable } \Longleftrightarrow
$$




$$
\forall \epsilon>0 \text { exists } \alpha \text { such that } d(0)<\alpha \Rightarrow d(t)<\epsilon \quad \forall t>0 .
$$

Thus stability expresses the continuity of the solution of (1) with respect to the initial conditions at the equilibrium solution. An equilibrium is asymptotically stable if it is stable and if small perturbed motions satisfy in addition

$$
d(t) \rightarrow 0 \text { when } t \rightarrow+\infty
$$

\subsection{Linearization method and Liapunov's theorem}

The fact that perturbed evolutions must stay near the equilibrium suggests the introduction of the associated linearized equation

$$
\dot{y}^{*}=A y^{*} \text { with } A=F, y\left(y_{e}\right) .
$$

Linearization consists of replacing $F(y)$ by its first-order expansion $F\left(y_{e}\right)+F, y_{y}\left(y_{e}\right) \cdot\left(y-y_{e}\right)$ at the equilibrium position by posing $y^{*}=y-y_{e}$. The linearized equation is a system of linear differential equations of order 1 with constant coefficients. This equation has as a general solution

$$
y^{*}(t)=\Re\{C \exp t A\}, \quad C \in \mathbf{C}^{n} .
$$

A more explicit expression of this general solution can be obtained in terms of the eigenvalues of the matrix $A$

$$
(A-s I) X=0 .
$$

Eigenvalues $s$ are real or conjugated complex roots of the characteristic equation

$$
\operatorname{det}(A-s I)=0
$$

since $A$ is a real matrix. From the Jordan decomposition of a matrix, it is classically established that the following expressions are obtained for the components of the linearized solution $y^{*}$

$$
y_{j}^{*}=\Re\left\{\sum_{i=1}^{N} P_{j}^{i}(t) \exp s_{i} t\right\}
$$

where $N$ is the number of distinct eigenvalues, $P_{j}^{i}(t)$ is a polynomial in $t$, of degree $\beta_{i}-1$ at most, $\beta_{i}$ is the dimension of the characteristic sub-space of $A$ associated with the eigenvalue $s_{i}$. In particular, in the case of simple eigenvalues $\beta_{i}=1 \forall i$.

Thus, it follows that $y^{*} \rightarrow 0$ when $t \rightarrow+\infty$ if $\Re\left(s_{i}\right)<0 \forall i$ and $y^{*} \rightarrow+\infty$ when $t \rightarrow+\infty$ if there exists at least one index $i$ such that $\Re\left(s_{i}\right)>0$.

Liapunov's theorem This conclusion about the linearized equation leads also to general results on the actual equation through the theorem

Liapunov's theorem The following fundamental results hold:

- If $\Re\left(s_{i}\right)<0$ for all $i$, the considered equilibrum is asymptotically stable.

- If there exists an index $i$ such that $\Re\left(s_{i}\right)>0$, the equilibrium is unstable. 
- If $\Re\left(s_{i}\right) \leq 0$ for all $i$ with at least one index $j$ such that $\Re\left(s_{j}\right)=0$, no conclusion is available for this uncertain situation. In this case, the linearization does not suffice and the conclusion depends on higher order terms i.e. on the nonlinear part of $F$.

Indeed, if $\Re\left(s_{i}\right)<0$ for all $i$, there exist two positive constants $c$ and $p$ such that

$$
\|\exp t A\| \leq c \exp -p t \text { for all } t \geq 0
$$

Let $r$ be the nonlinear part of $F$

$$
F(y)=F\left(y_{e}\right)+A\left(y-y_{e}\right)+r .
$$

A constant $\alpha$ can always be found such that

$$
\left\|y-y_{e}\right\| \leq \alpha \Rightarrow\|r\| \leq \frac{p}{2 c}\left\|y-y_{e}\right\| .
$$

Let $d(o)=\left\|y(0)-y_{e}\right\| \leq \alpha_{o}=\frac{\alpha}{2 c}$. A solution starting from an initial value $y(0)$, will remain inside a sphere of radius $\alpha$. Indeed, in the opposite case, there exists by continuity a time $t_{1}$ such that

$$
d\left(t_{1}\right)=\alpha, \quad d(s)<\alpha \forall 0<s<t_{1} .
$$

The general expression of the solution

$$
y(t)-y_{e}=\left(y(0)-y_{e}\right) \exp t A+\int_{0}^{t} \exp (t-s) A r\left(y(s), y_{e}\right) d s
$$

gives then the inequality

$$
\begin{gathered}
d\left(t_{1}\right) \leq c \alpha_{o} \exp -p t_{1}+c \int_{0}^{t_{1}} \exp -p\left(t_{1}-s\right) \frac{p}{2 c} d(s) d s \\
=\frac{\alpha}{2} \exp -p t_{1}+\frac{\alpha}{2}\left(1-\exp -p t_{1}\right)=\frac{\alpha}{2}
\end{gathered}
$$

which is impossible. Thus, it is concluded that $d(t)<\alpha$ for all $t>0$ if $d(o) \leq \frac{\alpha}{2 c}$. It follows that

$$
\forall t \geq 0, d(t) \exp p t \leq c \alpha_{o}+\frac{p}{2} \int_{0}^{t} d(s) \exp p s d s .
$$

This inequality can also be written as

$$
\dot{\phi}(t)-\frac{p}{2} \phi(t) \leq c \alpha_{o}
$$

with $\phi(t)=\int_{0}^{t} \exp p s d(s) d s$. After multiplication by $\exp -\frac{p}{2} t$ and after integration on $[0, t]$, it follows that (Gronwall's lemma)

$$
\phi(t) \leq \frac{2 c \alpha_{0}}{p}\left(\exp \frac{p}{2} t-1\right)
$$


This inequality leads to

$$
\dot{\phi}(t) \leq c \alpha_{o} \exp \frac{p}{2} t
$$

which gives

$$
\forall t \geq 0, d(t) \leq c \alpha_{o} \exp -\frac{p}{2} t .
$$

It is concluded that the considered equilibrium is asymptotically stable.

If there exist an index $i$ such that $\Re\left(s_{i}\right)>0$, then the equilibrium is necessarily unstable. The reader can refer to classical textbooks such as Coddington and Levinson (1955), Hale and Kocak (1991) for a proof of this result.

Divergence and flutter instabilities The linearization method can be applied in a simple way for mechanical systems. It is not necessary to make the theoretical change of variable to obtain a system of first order equations. The system of second order equations can be directly linearized. Thus, for a discrete mechanical system defined by $m$ free parameters $q_{i}$, the fundamental law of dynamics and constitutive laws lead to the system of second order differential equations

$$
\frac{d}{d t} C,,_{\dot{q}}-C,,_{q}=Q(q, \dot{q})
$$

where $C(q, \dot{q})$ denotes the kinetic energy and $Q(q, \dot{q})$ the generalized internal and external force. Linearized equations of motion near an equilibrium state $q^{e}$ are:

$$
\mathbf{M} \ddot{q}^{*}+\mathbf{N} \dot{q}^{*}+\mathbf{K} q^{*}=0
$$

where the matrices $\mathbf{M}, \mathbf{N}, \mathbf{K}$ are related to the kinetic energy $C(q, \dot{q})$ and the generalized force $Q(q, \dot{q})$ by

$$
\mathbf{M}=\frac{\partial^{2} C}{\partial \dot{q} \partial \dot{q}}\left(q^{e}, 0\right), \quad \mathbf{N}=-\frac{\partial Q}{\partial \dot{q}}\left(q^{e}, 0\right), \quad \mathbf{K}=-\frac{\partial Q}{\partial q}\left(q^{e}, 0\right),
$$

and represent respectively the mass, damping and stiffness matrices. The mass matrix $\mathbf{M}$ is symmetric and positive-definite while matrices $\mathbf{K}, \mathbf{N}$ are a priori only arbitrary square matrices. The generalized eigenvalue problem

$$
\left(s^{2} \mathbf{M}+s \mathbf{N}+\mathbf{K}\right) X=0 .
$$

is then introduced. Eigenvalues $s$ are roots of the polynomial equation

$$
\operatorname{det}\left(s^{2} \mathbf{M}+s \mathbf{N}+\mathbf{K}\right)=0
$$

and lead to Liapunov's theorem with the same statement:

-If there exists an index $i$ with $\Re\left(s_{i}\right)>0$ and $\Im\left(s_{i}\right) \neq 0$, the considered equilibrium is unstable by Liapunov's theorem. A small perturbation may lead to a growing perturbed motion following the associated mode. This motion is an oscillating motion with increasing amplitude. By definition it is said that flutter instability occurs.

-If there exists an index $i$ such that $\Re\left(s_{i}\right)>0$ and $\Im\left(s_{i}\right)=0$, the equilibrium is unstable. A perturbed motion will grow without oscillation and it is said that there is divergence instability. 

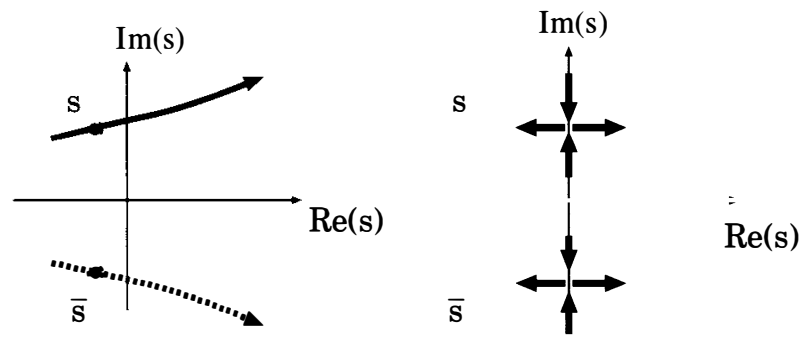

Figure 3: Flutter instability. For a system depending on a load parameter, the loss of stability by flutter occurs when an eigenvalue goes across the imaginary axis or when there is confusion between two close purely imaginary eigenvalues (i.e. confusion of two close frequencies).

In practical applications, the evolution equation (1) is often considered with a function $F$ depending on a load parameter $\lambda$

$$
\dot{y}=F(y, \lambda) .
$$

In this case, the possible equilibrium positions are solutions of the equation $F(y, \lambda)=0$. This equation defines implicitly the equilibrium response $y=y(\lambda)$ of the system as a function of $\lambda$. By linearization, the matrix $A(\lambda)=F, y(y(\lambda), \lambda)$ depends on $\lambda$. The graph of eigenvalues of $A$ as a function of the load parameter $s_{i}=s_{i}(\lambda)$ in the complex plane must be considered in the application of Liapunov's theorem. For stable equilibria, these curves must belong to the left of the imaginary axis. There is a transition to instability when one eigenvalue (and its conjugate) goes across the imaginary axis or when two eigenvalues are confused on the imaginary axis as shown in Figure 3 and Figure 4.

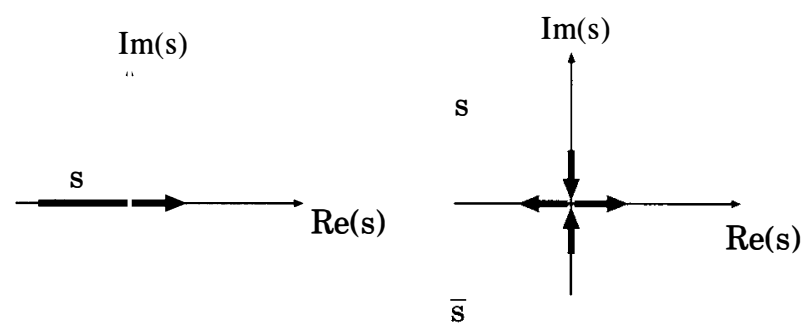

Figure 4: Divergence instability. Loss of stability due to divergence occurs when a real eigenvalue goes across the imaginary axis or when two conjugate and purely imaginary eigenvalues vanish (i.e. nullity of a vibration frequency).

\subsection{Hopf bifurcation}

It is assumed that a fundamental equilibrium curve $y=y_{o}(\lambda)$ of a system governed by equation (1) exists and that for $\lambda=\lambda_{c}$, there is flutter instability. More precisely, it is assumed that $A(\lambda)$ 
has a pair of conjugate complex eigenvalues $s_{ \pm}=\alpha(\lambda) \pm i \beta(\lambda)$ such that

$$
\alpha\left(\lambda_{c}\right)=0, \beta\left(\lambda_{c}\right)=\beta_{c}>0
$$

and that $\alpha_{c}^{\prime}=\frac{d \alpha}{d \lambda}\left(\lambda_{c}\right)$ satisfies the condition

$$
0<\left|\alpha_{c}^{\prime}\right|<+\infty
$$

This means that this pair of eigenvalues goes across the imaginary axis in a transverse way. All other eigenvalues are assumed to have strictly negative real parts.

Hopf's theorem Under these assumptions, the following results hold:

- The considered critical point is a bifurcation point of the static curve to a curve of periodic responses of amplitude increasing generically as $\left|\lambda-\lambda_{c}\right|^{\frac{1}{2}}$.

- The bifurcated periodic response is orbitally and asymptotically stable near the the critical point if $\alpha_{c}^{\prime} \lambda_{2}>0$ and unstable if $\alpha_{c}^{\prime} \lambda_{2}<0$.

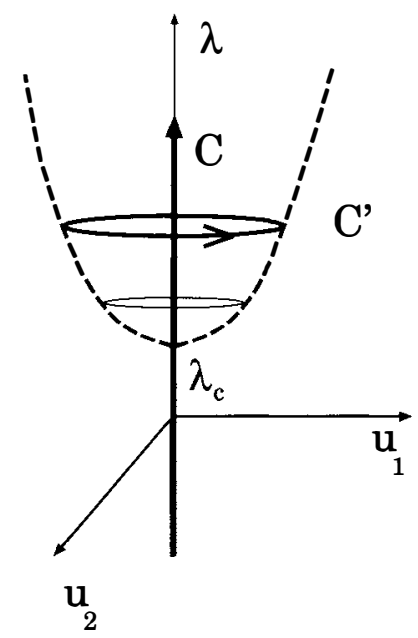

Figure 5: Hopf's bifurcation.

The reader may refer to various works, for example to Iooss and Joseph (1981) or to Marsden and McCracken (1976) for a complete proof of Hopf's theorem. The bifurcated response can be obtained by the method of asymptotic expansion. The change of variable $\tau=\frac{2 \pi}{T} t$ gives the possibility to consider only periodic functions $Y(\tau)$ defined on the interval $[0,2 \pi]$ with $Y(\tau)=$ $y\left(\frac{T}{2 \pi} \tau\right)$. Thus, the evolution equation can be written as

$$
\omega \dot{Y}=F(Y, \lambda)
$$

where $\omega=\frac{2 \pi}{T}$ and $\dot{Y}=\frac{d Y}{d \tau}$. The bifurcation of the static curve $y=y_{o}(\lambda)$ to a dynamic periodic response at a critical value $\lambda_{c}$ can be defined by the asymptotic expansion of the bifurcated 
response at the vicinity of $Y_{o}\left(\lambda_{c}\right)$

$$
\left\{\begin{array}{l}
\lambda=\lambda_{c}+\lambda_{1} \xi+\lambda_{2} \frac{1}{2} \xi^{2}+\cdots \\
\omega=\omega_{0}+\omega_{1} \xi+\omega_{2} \frac{1}{2} \xi^{2}+\cdots \\
Y=Y_{o}(\lambda)+Y_{1} \xi+Y_{2} \frac{1}{2} \xi^{2}+\cdots
\end{array}\right.
$$

where $Y_{i}(\tau)$ are $2 \pi$-periodic functions. By an identification of terms of different orders in $\xi$, this expansion can be obtained step by step. The stability of the bifurcated response can be easily understood as a straightforward extension of the concept of stability of an equilibrium to an evolution. It is relative to the question of orbital stability. A rigorous proof of this result can be found in various works, for example in Iooss and Joseph (1981) or in Marsden and McCracken (1976).

Hopf's bifurcation and the stability analysis near the critical point can be easily understood from the reduction of the differential system into a pair of differential equations by the concept of a center manifold, cf. for example Troger and Steindl (1991), Hale and Kocak (1991), Nguyen (2000). The method of reduction by the center manifold plays for dynamic problems a similar role as the Liapunov-Schmidt's method for static problems of elasticity. If $X=X_{1}+i X_{2}$ denotes the eigenvector associated with the eigenvalue $s=i \beta_{c}$, the considered system of differential equations is written in the form

$$
\begin{gathered}
y=u+w, \quad u \in R_{c}, \quad w \in R_{s}, R_{c} \oplus R_{s}=\mathbf{R}^{n}, \\
\dot{u}=G_{c}(u, w, \lambda), \quad \dot{w}=G_{s}(u, w, \lambda)
\end{gathered}
$$

where $R_{c}$ is the invariant space spanned by the vectors $X_{1}, X_{2}$. The center manifold method states that there exists a reduction defined by

$$
w=h(u, \lambda) \text { with } h,_{u}\left(0, \lambda_{c}\right)=0
$$

such that the dynamic behaviour of the motions is also given by the reduced system of differential equations

$$
\dot{u}=G_{c}(u, h(u, \lambda), \lambda) .
$$

The hypersurface $M_{\lambda}$, defined by equation $w=h(u, \lambda)$ for fixed $\lambda$, is by definition the center manifold. This surface is an attracting surface for all nearby motions, thus the stability behaviour is completely governed by the motions on the center manifold. With the notation

$$
u=x_{1} X_{1}+x_{2} X_{2}, x=\left(x_{1}, x_{2}\right),
$$

the reduced equation to be considered is

$$
\dot{x}=\left[\begin{array}{cc}
\alpha(\lambda) & \beta(\lambda) \\
-\beta(\lambda) & \alpha(\lambda)
\end{array}\right] x+f(x, \lambda) \text { with } f\left(0, \lambda_{c}\right)=0, f,,_{x}\left(0, \lambda_{c}\right)=0 .
$$

This system can be written in polar coordinates $x_{1}=r \cos \theta, x_{2}=-r \sin \theta$ as

$$
\frac{d r}{d \theta}=R(r, \theta, \lambda), \dot{\theta}=\beta_{c}(1+\Theta(r, \theta, \lambda))
$$

where $R$ and $\Theta$ are $2 \pi$-periodic functions with respect to $\theta$ with $R\left(0, \theta, \lambda_{c}\right)=0,|\Theta|<1$, cf. Hale and Kocak (1991). Thus the dynamic motions encircling the origin can be discussed from the first equation. 
Example 1 The example of a system with two degrees of freedom, governed by two differential equations

$$
\left\{\begin{array}{l}
\frac{d y_{1}}{d t}=-y_{2}+y_{1}\left(\lambda-y_{1}^{2}-y_{2}^{2}\right), \\
\frac{d y_{2}}{d t}=y_{1}+y_{2}\left(\lambda-y_{1}^{2}-y_{2}^{2}\right) .
\end{array}\right.
$$

is considered. In polar coordinates, these equations are

$$
\frac{d r}{d t}=r\left(\lambda-r^{2}\right), \quad \frac{d \theta}{d t}=1 .
$$

The trivial equilibrium $y_{o}(\lambda)=(0,0) \forall \lambda$ is stable for $\lambda<0$ and $\lambda_{c}=0$ is the critical bifurcation load. This equilibrium is also stable at $\lambda=\lambda_{c}$ since the perturbed motions of this equilibrium are governed by the equations

$$
\frac{d r}{d t}=-r^{3}, \quad \frac{d \theta}{d t}=1 .
$$

The bifurcated response $(\theta=t, r=\sqrt{\lambda})$ is stable for $\lambda>0$. In this example, $\alpha(\lambda)=\lambda$ and $\beta_{c}=1$. From a given initial condition and for a given value of $\lambda$, the evolution of the system is defined by a trajectory in the plane $\left(y_{1}, y_{2}\right)$. This trajectory tends to the origin $\mathrm{O}$ when $t \rightarrow \infty$ if $\lambda<0$. The origin $\mathrm{O}$ is an attractive point in this condition. If $\lambda>0$, this point becomes repulsive and the trajectory tends to the circle $r=\sqrt{\lambda}$ which is attractive.

Example 2 The example of a simple oscillator is considered again. It is assumed here that the friction force is related to the relative sliding velocity by a viscous law

$$
f=\lambda(\dot{x}-w)+a(\dot{x}-w)^{3} .
$$

The dynamic equation is

$$
\left.M \ddot{x}-\lambda(\dot{x}-w)-a(\dot{x}-w)^{3}\right)+K x=0 .
$$

The static response is

$$
x_{o}(\lambda)=-\frac{w}{K}\left(\lambda+a w^{2}\right) .
$$

The stability of this equilibrium is obtained from the characteristic equation

$$
s^{2} M-s\left(\lambda+3 a w^{2}\right)+K=0,
$$

thus

$$
s=\frac{\lambda+3 a w^{2} \pm \sqrt{\left(\lambda+3 a w^{2}\right)^{2}-4 K M}}{2 M} .
$$

A pair of conjugate eigenvalues goes across the imaginary axis at the critical load $\lambda_{c}=-3 a w^{2}$ with $\alpha_{c}^{\prime}=1 / 2 M>0$ and $\beta=\sqrt{K / M}$. The transversality assumption is thus fulfilled and there is Hopf's bifurcation. The bifurcated response can be obtained directly from the second order differential equation (25). The $2 \pi$-periodic function $Y(\tau)$, defined by $\tau=\omega t$ and $Y(\tau)=$ $x(t(\tau))$, must satisfy

$$
\left.M \omega^{2} Y^{\prime \prime}-\lambda\left(\omega Y^{\prime}-w\right)-a\left(\omega Y^{\prime}-w\right)^{3}\right)+K Y=0 .
$$


The previous asymptotic expansion (18) is now inserted into the equation (26) in order to derive the governing equations for different orders of $\xi$. The results $\lambda_{1}=\omega_{1}=0$ are also anticipated in order to simplify the calculations. It follows that the equation of order 1 is

$$
M \omega_{o}^{2} Y_{1}^{\prime \prime}+K Y_{1}=0
$$

thus $\omega_{o}=\sqrt{K / M}$ and $Y_{1}=C \sin (\tau+\varphi)$. It is sufficient to consider the phase $\varphi=0$. The constant $C$ must be 1 in order to have $\xi$ as an initial amplitude. The equation of order 2 is

$$
M \omega_{o}^{2} Y_{2}^{\prime \prime}+K Y_{2}+6 a w \omega_{o}^{2} \cos ^{2} \tau=0,
$$

and gives $Y_{2}=-\frac{a w}{M}(3-\cos 2 \tau)$. The equation of order 3 is

$$
\begin{gathered}
M \omega_{o}^{2} Y_{3}^{\prime \prime}+K Y_{3}-\omega_{2} 6 \sqrt{K M} \sin \tau-\lambda_{2} \omega_{o} \cos \tau \\
-6 a \omega_{o}^{3} \cos ^{3} \tau-9\left(\frac{a w}{M}\right)^{2} K \sin 2 \tau \cos \tau=0,
\end{gathered}
$$

and gives

$$
\omega_{2}=-3 \omega_{o}\left(\frac{a w}{M}\right)^{2}, \quad \lambda_{2}=-\frac{3 a K}{2 M}, \quad Y_{3}=\frac{3 a \omega_{o}}{16 M} \cos 3 \tau-\left(\frac{3}{2} \frac{a w}{M}\right)^{2} \sin 3 \tau .
$$

Finally, the trivial response $x=x_{o}(\lambda)$ is asymptotically stable for $\lambda<\lambda_{c}$ and unstable for $\lambda>\lambda_{c}$. The bifurcated response is

$$
\begin{gathered}
x(t)=-\frac{w}{K}\left(\lambda(\xi)+a w^{2}\right)+\xi \sin \omega t-\frac{1}{2} \xi^{2} \frac{a w}{M}(3-\cos 2 \omega t) \\
+\xi^{3}\left(\frac{a \omega_{o}}{32 \bar{M}} \cos 3 \omega t-\frac{3}{8}\left(\frac{a w}{M}\right)^{2} \sin 3 \omega t\right), \\
\omega=\frac{2 \pi}{T}=\sqrt{\frac{K}{M}}\left(1-\frac{3}{2}\left(\frac{a w}{M}\right)^{2} \xi^{2}\right)+0\left(\xi^{3}\right), \\
\lambda=-3 a w^{2}-\frac{3 a K}{4 M} \xi^{2}+\ldots
\end{gathered}
$$

Since $\alpha_{c}^{\prime} \lambda_{2}$ has the sign of $-a$, the periodic response is unstable for $a>0$ and asymptotically stable for $a<0$.

However, if the friction force obeys Coulomb's law, then the analytical calculation cannot be done in the same spirit since the dynamic equations are not regular. The proof of Hopf's theorem cannot be applied and the conclusion is no more valid. The results presented in the introduction have been derived in a different way.

Example 3 The example of a three-dimensional oscillator composed of a mass attached to three springs as shown in Figure 6, in contact with a moving half-space and submitted to a given normal force, is considered. Let $u_{T}$ denote the plane displacement of the mass, $k_{N N}, k_{N T}$ and $k_{T T}$ the sub-rigidity matrices associated with normal and in-plane displacements. Then, it was establihed that the condition $f<f_{p}$ defined by

$$
1-f_{p} k_{N T} k_{T T}^{-1}[\tau]=0
$$


where $\tau=\frac{w}{\|w\|}$, ensures the existence and the uniqueness of the equilibrium.

It is already said that Hopf's theorem cannot be applied to the system of dynamic equations when Coulomb's law of friction is assumed because unilateral contact and Coulomb's equations are not differentiable conditions. However, if the assumption of slip regime is accepted for all time, then this theorem can be applied. This is for example the case when the translation velocity of the obstacle is very high.

Let $n$ be the normal direction to $\tau$ and oriented such that $\tau \cdot k_{T T}[n]>0$ when $\tau \cdot k_{T T}[n] \neq 0$. Then, it is established that, cf. Moirot (1998)

$$
\begin{gathered}
\tau \cdot k_{T T}[n]=0 \Rightarrow \text { stable equilibrium. } \\
\tau \cdot k_{T T}[n]-f k_{N T}[n]>0 \Rightarrow \text { asymptotically stable equilibrium. } \\
\tau \cdot k_{T T}[n]-f k_{N T}[n]<0 \Rightarrow \text { unstable equilibrium with flutter instability. }
\end{gathered}
$$

There is a Hopf bifurcation in the last case. It is concluded that, under the assumption of slip regime for all time, there exists a critical value of the friction coefficient $f_{c d}$ :

$$
\begin{gathered}
f_{c d}=\infty \text { if } \tau \cdot k_{T T}[n]=0 \text { or if } k_{N T}[n]<0, \quad \tau \cdot k_{T T}[n]>0 ; \\
\text { and } \quad f_{c d}=\frac{\tau \cdot k_{T T}[n]}{k_{N T}[n]} \text { if } k_{N T}[n]>0
\end{gathered}
$$

such that the steady sliding solution is unstable if $f>f_{c d}$.

\subsection{Standard dissipative systems}

Coulomb's friction has often been interpreted in the literature as a non-associated law. Indeed, the velocity $\left(v_{T}, v_{N}\right)$ is not a normal to the domain of admissible forces $-R=-(T, N)$. An equivalent interpretation also exists by saying that the normality law is satisfied by the flux $v_{T}$ and the force $-T$, but the set of admissible forces, which is a sphere of radius $f N$, depends on the present state through the present value of $N$. Thus, contact under friction is a dissipative phenomenon with a state-dependent dissipation potential. Contact with friction is a particular class of standard dissipative systems. The dissipation by Coulomb's dry friction per unit surface is

$$
d=-T \cdot v_{T}=f N\left\|v_{T}\right\| .
$$

The friction force can also be written in terms of a dissipation potential

$$
-T=\frac{\partial D}{\partial v_{T}} \text { with } D\left(v_{T}, N\right)=f N\left\|v_{T}\right\| .
$$

It is recalled that the notions of state variables, state equations, energy potential, dissipation potential and normality laws offer a general framework for the study of solids denoted as the standard dissipative systems. General results obtained in plasticity have been extended for such a system, cf. Nguyen (2000). 

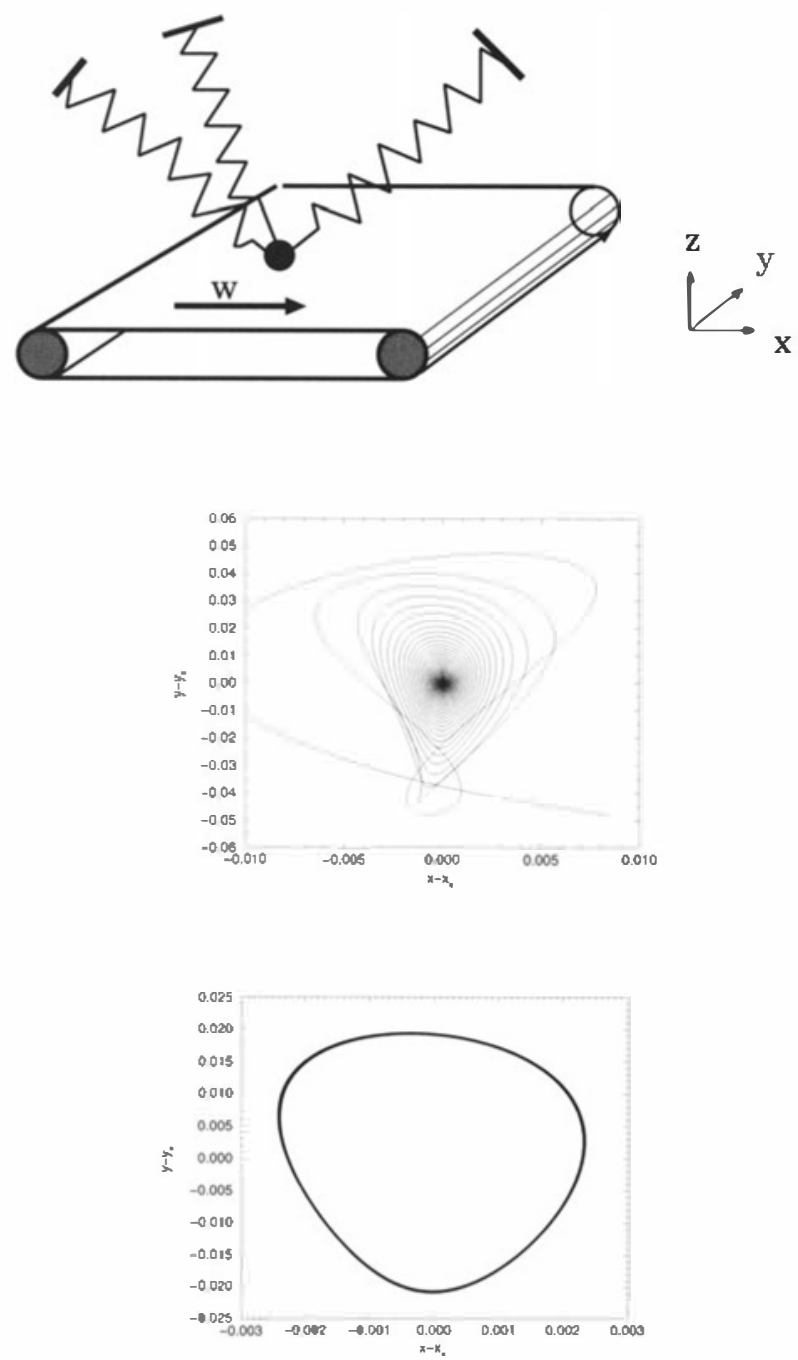

Figure 6: Equilibrium and stability of a three-dimensional mass-spring system in contact with a moving half-space, after Moirot, 1998. The translation motion of the obstacle is assumed here to be great enough in order to ensure for all times the sliding regime of the mass. The stationary sliding motion is asymptotically stable for $f=0.21$. The convergence to the equilibrium position of a small perturbed motion is shown in the first graph. For $f=0.226$, the stationary sliding motion is unstable and the perturbed motion tends to a limit cycle after Hopf 's theorem as shown in the second graph. 
Standard systems Consider an arbitrary system defined by variables $q$ such that the governing equation can be written in the spirit of the virtual work equation as

$$
\delta W_{i}+\delta W_{e}=0 \quad \forall \quad \delta q, \quad \delta W_{i}=Q_{i n t} \cdot \delta q, \quad \delta W_{e}=F(t) \cdot \delta u
$$

where $F(t)$ is a given function and

$$
Q_{\text {int }}=Q_{i n t}(q, \dot{q})
$$

by assumption. It is assumed that there exist an energy potential $\mathbf{W}(q)$ and a dissipation potential $\mathbf{D}(\dot{q}, q)$ such that the following expression holds

$$
Q_{\text {int }}(q, \dot{q})=-\mathbf{W}_{,_{q}}-\mathbf{D},_{\dot{q}} .
$$

Thus, the internal forces are composed of conservative forces derived from the potential $\mathbf{W}(q)$ and of dissipative forces derived from the viscous potential $\mathbf{D}(\dot{q}, q)$. Under this assumption, the governing equations can also be written as

$$
\mathbf{W},{ }_{q}+\mathbf{D}, \dot{q}-F=0, \quad q(0)=q^{0}
$$

in a quasi-static transformation from an initial state $q^{0}$. The energy potential of the system is

$$
\mathbf{E}(q, t)=\mathbf{W}(q)-F(t) \cdot u
$$

and Biot's equation is the governing equation of the system in a quasi-static evolution

$$
\mathbf{E},_{q}+\mathbf{D},_{\dot{q}}=0, \quad q(0)=q^{0} .
$$

For the sake of clarity, it is assumed that $F$ depends on $t$ via a control parameter $\lambda$ which is a given function on time $F=F(\lambda(t))$. In this case, the energy potential is $\mathbf{E}(q, \lambda)$. More generally, if $F=F(q, \lambda)=-\mathbf{P},_{q}(q, \lambda)$, then the energy potential is $\mathbf{E}(q, \lambda)=\mathbf{W}(q)+\mathbf{P}(q, \lambda)$, thus the case of time-dependent conservative external forces can also be included in the description.

If $q=(u, \alpha)$ where $u$ denotes the displacement components and $\alpha$ the internal parameters, a dynamic evolution of the system can be described by the governing equation

$$
\delta W_{i}+\delta W_{e}+\delta W_{j}=0, \quad \delta W_{j}=-J(\ddot{u}, \dot{u}, u) \cdot \delta u,
$$

It follows that the dynamic equations are

$$
J+\mathbf{E}_{,_{u}}+\mathbf{D},_{\dot{u}}=0, \quad \mathbf{E}_{\alpha}+\mathbf{D},_{\dot{\alpha}}=0, \quad q(0)=q^{0}, \quad \dot{u}=v^{0} .
$$

By definition, such a system is denoted as a standard dissipative system. Standard dissipative systems are governed in a quasi-static evolution by Biot's differential equation (33) and in a dynamic evolution by the second order differential equation (35).

If $u$ is a reversible variable then $\mathbf{D}=\mathbf{D}(\dot{\alpha}, u, \alpha)$. In this case, the governing equations of a quasi-static evolution can also be written as

$$
\mathbf{E},_{u}=0, \quad \mathbf{E},_{\alpha}+\mathbf{D}, \dot{\alpha}=0, \quad \alpha(0)=\alpha^{0}
$$


or, in an equivalent way as the system of equations

$$
\mathbf{E}_{,}=0, \quad A=-\mathbf{E},_{\alpha}, \quad \dot{\alpha}=\mathbf{D}^{*},_{A}, \quad \alpha(0)=\alpha^{0} .
$$

In particular, for a conservative system, as in the case of an elastic solid under time-dependent conservative loads, the governing equation $\mathbf{E},_{u}=0$ is obtained as a particular case.

Dry friction and plasticity are time-independent irreversible processes. A time-independent mechanism of irreversibility is associated with a particular dissipative potential which is convex and positively homogeneous of degree 1 with respect to $\dot{\alpha}$

$$
\mathbf{D}(m \dot{\alpha}, \alpha)=m \mathbf{D}(\dot{\alpha}, \alpha) \quad \forall m>0 .
$$

Such a function is not differentiable at $\dot{\alpha}=0$, but sub-differentiable. The set $\mathbf{C}$ of sub-gradients at this point

$$
\mathbf{C}=\partial_{\dot{\alpha}} \mathbf{D}(0, \alpha)=\left\{A^{*} \mid A^{*^{\prime}} \cdot \delta \alpha \leq \mathbf{D}(\delta \alpha, \alpha) \forall \delta \alpha\right\}
$$

is the convex domain of admissible forces. The dual dissipation potential is in this case the indicator function of the domain of admissible forces. The force-flux relationship $\dot{\alpha}=\mathbf{D}^{*},{ }_{A}$ can be written in the form of the normality law

$$
\dot{\alpha}=\mathbf{N}_{\mathbf{C}}(A)
$$

which states that $\dot{\alpha}$ must be an external normal to the admissible domain at the present state of the force $A$. It is well known that this law of evolution can also be equivalent to the maximum dissipation principle which is classical in plasticity and known as the principle of maximum plastic work

$$
\mathbf{D}(\dot{\alpha}, \alpha)=\max _{A^{*} \in C} A^{*} \cdot \dot{\alpha} .
$$

Quasi-static evolution of time-independent standard systems The governing equations (37) for a quasi-static evolution of a time-independent standard system can be written as

$$
\mathbf{E}_{,_{u}}=0, \quad A=-\mathbf{E},_{\alpha}, \dot{\alpha}=\mathbf{N}_{\mathbf{C}}(A), \quad \alpha(0)=\alpha^{0} .
$$

Since, from the maximum dissipation principle

$$
\mathbf{D}(\delta \alpha)-A \cdot \delta \alpha \geq \mathbf{D}(\dot{\alpha})-A \cdot \dot{\alpha}=0 \quad \forall \delta \alpha,
$$

the governing equations can also be written in the form of a variational inequality

$$
\mathbf{E}_{, u} \cdot(\delta u-\dot{u})+\mathbf{E}_{, \alpha} \cdot(\delta \alpha-\dot{\alpha})+\mathbf{D}(\delta \alpha, \alpha)-\mathbf{D}(\dot{\alpha}, \alpha) \geq 0 \quad \forall(\delta u, \delta \alpha) .
$$

The normality law is an incremental law. Indeed, if $\gamma(t)$ and $G(t)$ denote two histories of flux and force associated by the normality law, then the flux $\gamma(t)$ and the force rate $\dot{G}(t)$ are related by the following proposition

Proposition 1. Let $\gamma(t)$ and $G(t)$ be a flux and a force associated by the normality law with a convex $\mathbf{C}$ of non-void interior, depending on a given function $y(t)$ for $t \in[0, T]$. If the rates 
$\dot{y}(t), \gamma(t)$ and $\dot{G}(t)$ are piecewise-continuous then the following expressions hold for the righthand-side (r.h.s.) rates

$$
\begin{gathered}
-\dot{G} \cdot \gamma+\dot{y} \cdot \mathbf{D},_{y}(\gamma, y)=0 \\
-\dot{G} \cdot \gamma^{*}+\dot{y} \cdot \mathbf{D},_{y}\left(\gamma^{*}, y\right) \geq 0 \quad \forall \gamma^{*} \text { admissible }
\end{gathered}
$$

by definition

$$
\gamma^{*} \text { admissible } \Leftrightarrow \gamma^{*} \in \mathbf{N}_{\mathbf{C}(y)}(G)
$$

The proof of this proposition follows simply from the maximum dissipation principle (41). To simplify the notation, let $\tau=t^{\prime}-t, G=G(t)$ and $G_{\tau}=G\left(t^{\prime}\right)$. For all $\gamma^{*} \in \mathbf{N}_{\mathbf{C}(y)}(G)$, it follows that

$$
g(\tau)=\mathbf{D}\left(\gamma^{*}, y_{\tau}\right)-G_{\tau} \cdot \gamma^{*} \geq 0, \quad g(0)=0
$$

thus the r.h.s. derivative $\dot{g}(0+)$ is non-negative and gives (45).

To obtain (44) at a time $t$ where $y(t)$ and $G(t)$ are differentiable, it is first observed that $\dot{g}(0)=\dot{g}(0+)=\dot{g}(0-)$ by assumption. Then the previous discussion shows that $\dot{g}(0+) \geq 0$ and $\dot{g}(0-) \leq 0$, thus it follows that $\dot{g}(0)=0$. Equation (44) then holds with $\gamma^{*}=\gamma$.

The same result is also valid at a point $t$ where $\dot{y}(t)$ or $\dot{G}(t)$ or $\gamma(t)$ are discontinuous, since it holds for $t^{\prime}>t$ and for the limit $t^{\prime} \rightarrow t$.

As a consequence of the proposition, it should be noted that, if the assumption of a stateindependent potential is satisfied, i.e. if the dissipation potential does not depend on the present state, then it is concluded that the r.h.s. rates satisfy $\dot{G} \cdot \gamma=0$. This orthogonality property is very classical in perfect plasticity.

The relations (44), (45), written for $y(t)=q(t)$, together with the equations $A=-\mathbf{E},_{\alpha}$ and $\mathbf{E},_{u}=0$, lead to the following description of the rate problem which consists of obtaining the rate response of the system $\dot{q}$ as a function of the rate data $\dot{\lambda}$ when the present state is assumed to be known:

Proposition 2. The rate response $\dot{q}$ is a solution of the variational inequality

$$
\begin{gathered}
\left(\mathbf{E},_{q q} \cdot \dot{q}+\mathbf{E},_{q \lambda} \cdot \dot{\lambda}\right) \cdot(\delta q-\dot{q})+\dot{q} \cdot\left(\mathbf{D},_{q}(\delta q, q)-\mathbf{D},_{q}(\dot{q}, q)\right) \geq 0 \\
\forall \delta q=(\delta u, \delta \alpha) \text { admissible. }
\end{gathered}
$$

This is a quadratic variational inequality defined in a convex set. This variational inequality is not necessarily symmetric due to the lack of symmetry of the term $\dot{q} \cdot\left(\mathbf{D},,_{q}(\delta q, q)\right.$ with respect to the pair $\dot{q}, \delta q$.

In particular, the uniqueness of the rate response $\dot{q}$ is ensured if the following positivity is satisfied for all admissible rates $\delta q_{1} \neq \delta q_{2}$

$$
\left(\delta q_{1}-\delta q_{2}\right) \cdot \mathbf{E},_{q q} \cdot\left(\delta q_{1}-\delta q_{2}\right)+\left(\delta q_{1}-\delta q_{2}\right) \cdot\left(\mathbf{D},_{q}\left(\delta q_{1}, q\right)-\mathbf{D},_{q}\left(\delta q_{2}, q\right)\right)>0
$$

The existence of a solution $\dot{q}$ is also ensured for all $\dot{\lambda}$ under the condition of positivity

$$
\mathbf{E},_{q q}\left[q^{*}, q^{*}\right]+q^{*} \cdot \mathbf{D},_{q}\left(q^{*}, q\right)>c\left\|q^{*}\right\|^{2} \forall q^{*} \text { admissible } \neq 0 .
$$

These conditions are however not optimal since it deals only with the symmetric part of the bilinear form $\dot{q} \cdot\left(\mathbf{D},,_{q}(\delta q, q)\right.$. 
As discussed by Hill (1958) in the framework of incremental plasticity, (48) furnishes a sufficient condition of static non-bifurcation and (49) a sufficient condition of static stability. It is well known that static stability also means dynamic stability in the particular cases of standard plasticity as well as of conservative systems, e.g. Nguyen (2000). These criteria are the basis of the theory of elastic and plastic buckling. But since the frictional contact problem is not symmetric, such a criterion can only have a limited interest in contact mechanics. The lack of symmetry is a source of difficulty as it is well known in non-conservative systems. The reader may refer to Klarbring (1990), Martins et al. (1999), Nguyen (2000) for some examples of non-existence, non-uniqueness in relation with stability analysis of a static evolution of solids in friction contact.

\section{Frictional equilibrium of a solid with a moving obstacle}

\subsection{Steady sliding of a solid on a half-space}

For the sake of simplicity, let us consider the problem of frictional equilibrium of an elastic solid in contact with a moving rigid half-space, in translation motion at constant velocity $w$ parallel to the free surface. In small deformation, the equilibrium position $u$ associated with the steady sliding solution must satisfy

$$
\int_{V} \nabla \delta u: L: \nabla u d V-\int_{S_{r}} r^{d} \cdot \delta u d a-\int_{S_{R}}\left(\delta u_{N} N+f N \tau \cdot \delta u_{T}\right) d a=0 .
$$

It is well known that this equation leads to a reduced form in terms of the displacement of the contact surface

$$
N=k_{N N}\left[u_{N}\right]+k_{N T}\left[u_{T}\right]+N^{d}, \quad T=f N \tau=k_{T N}\left[u_{N}\right]+k_{T T}\left[u_{T}\right]+T^{d} .
$$

The principal unknown $u_{N}$ must satisfy

$$
u_{N}=\mathbf{A}[N]+\mathbf{B}, \quad N \geq 0, u_{N} \geq 0, \quad N \cdot u_{N}=0
$$

with

$$
\begin{gathered}
\mathbf{A}=\left(k_{N N}-k_{N T} k_{T T}^{-1} k_{T N}\right)^{-1}\left(I-f k_{N T} h_{T N}\right) \\
h_{T N}[N]=k_{T T}^{-1}[N \tau], \quad \mathbf{B}=\left(k_{N N}-k_{N T} k_{T T}^{-1} k_{T N}\right)^{-1}\left[N^{d}-k_{N T} k_{T T}^{-1}\left[T^{d}\right]\right] .
\end{gathered}
$$

It is clear that the linear operator $\mathbf{A}$ is not symmetric if $f \neq 0$ :

$$
\left(N^{*}, \mathbf{A}[N]\right)=\int_{S_{R}} N^{*}(x) \mathbf{A}[N](x) d S \neq\left(N, \mathbf{A}\left[N^{*}\right]\right)
$$

A linear complementary problem (LCP) must be considered. In particular, the existence and uniqueness of a solution are ensured if $\mathbf{A}$ is positive-definite or P-positive, cf. Klarbring (1997), Cottle et al. (1992). 


\subsection{Stability of the steady sliding solution}

The stability of the steady sliding position can be obtained from the study of small perturbed motions. However, the equations of motion cannot be linearized without the assumption of an effective contact. Indeed, in the presence of a loose contact, a small perturbed motion is not necessarily governed by linear equations because of the possibility of separation and of the inherent nonlinearity of Coulomb's law. Under the assumption of an effective contact, if the sliding speed is never zero, the dynamic equations can be written as

$$
\begin{gathered}
\int_{V} \delta u \cdot \rho \ddot{u} d V+\int_{V} \nabla \delta u: L: \nabla u d V+\int_{S_{R}} N \delta u_{N} d S \\
+\int_{S_{R}} f N \frac{\dot{u}-w \tau}{\|\dot{u}-w \tau\|} \cdot \delta u_{T} d S=0 \forall \delta u, \delta N .
\end{gathered}
$$

Under this assumption of sliding motions, the linearization is possible and the linearized equations of motion are

$$
\begin{aligned}
& \int_{V} \delta u \cdot \rho \ddot{u}^{*} d V+\int_{V} \nabla \delta u: L: \nabla u^{*} d V+\int_{S_{R}} N^{*} \delta u_{N} d S \\
& +\int_{S_{R}} f\left\{\frac{N_{e}}{w}\left(\dot{u}_{T}^{*}-\left(\dot{u}_{T}^{*} \cdot \tau\right) \tau\right)+N^{*} \tau\right\} \cdot \delta u_{T} d S=0 \forall \delta u .
\end{aligned}
$$

with $u_{N}^{*}=0$ on $S_{R}$. After discretization, the equations of motion are

$$
\left\{\begin{array}{l}
U_{N}=0 \\
M_{Y Y} \ddot{Y}+K_{Y Y} Y=f \Phi(\dot{Y}) N+F_{Y} \\
M_{N Y} \ddot{Y}+K_{N Y} Y=N+F_{N}
\end{array}\right.
$$

where $u=\left(U_{N}, Y\right)$ and $\Phi(\dot{Y})$ is a matrix dependent on the direction of slipping. The linearized equations are

$$
\left\{\begin{array}{l}
U_{N}^{*}=0 \\
M_{Y Y} \ddot{Y}^{*}+K_{Y Y} Y^{*}=f \Phi_{N} N^{*}+f \Phi_{Y} \dot{Y}^{*}, \\
M_{N Y} \ddot{Y}^{*}+K_{N Y} Y^{*}=N^{*} .
\end{array}\right.
$$

The general expression $u^{*}=e^{s t} U$ with $U=\left(U_{N}=0, X\right)$ then leads to

$$
s^{2} M_{Y Y} X+K_{Y Y} X=f s \Phi_{Y} X+f \Phi_{N} \mathcal{N}, s^{2} M_{N Y} X+K_{N Y} X=\mathcal{N}
$$

or to the generalized eigenvalue problem

$$
s^{2}\left(M_{Y Y}-f \Phi_{N} M_{N Y}\right) X-s f \Phi_{Y} X+\left(K_{Y Y}-f \Phi_{N} K_{N Y}\right) X=0 .
$$

When the analysis is restricted to sliding motions, the considered equilibrium is asymptotically stable if $\Re(s)<0$ for all $s$ and unstable if there exists at least one value $s$ such that $\Re(s)>0$. This generalized eigenvalue problem can be written as $\left(s^{2} \bar{M}+s \bar{C}+\bar{K}\right) X=0$ with nonsymmetric matrices $\bar{M}, \bar{K}$ and complex eigenvalues and eigenvectors. This analysis leads to the definition of a critical value $f_{d} \geq 0$ such that the considered equilibrium is unstable when $f>f_{d}$.

To obtain the stability of the considered equilibrium, it is necessary to consider arbitrary perturbed motions taking account of the possibility of sticking, slipping and separation regimes. Such a complete justification is still lacking in the literature. 


\subsection{Instability of the steady sliding solution in the contact of two elastic layers}

The simple example of the frictional contact of two elastic infinite layers is considered here as an illustrating example. This problem was discussed analytically by Adams (1995) and Martins et al. (1995).

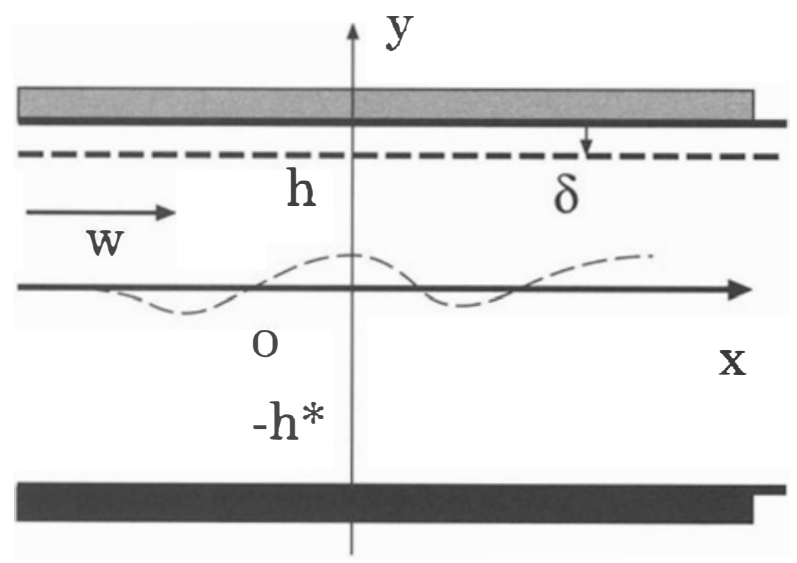

Figure 7: Contact with friction of two elastic layers

Governing equations and the steady sliding solution The contact in plane strain with friction of two infinite elastic layers, of thickness $\hat{h}$ and $\hat{h}^{*}$ respectively as shown in Figure 7, is considered. The lower face of the bottom layer is maintained fixed in the axes Oxyz. The upper face of the top layer, assumed to be in translation motion in the direction $O x$ of velocity $\hat{w}$, is compressed to the bottom layer by an implied displacement $\hat{\delta}<0$. At the interface $y=0$, the contact is assumed to obey Coulomb's law of friction with a constant friction coefficient. In terms of nondimensional variables defined by

$$
\begin{gathered}
c_{1}=\sqrt{(\lambda+2 \mu) / \rho}, \quad c_{2}=\sqrt{\mu / \rho}, \quad \tau=c_{2} / c_{1}, \\
x=\hat{x} / l, \quad y=\hat{y} / l, \quad h=\hat{h} / l, \quad \delta=\hat{\delta} / l, \\
w=\hat{w} / c_{2}, \quad t=\hat{t} c_{2} / l, \quad(\hat{t} \text { is the physical time })
\end{gathered}
$$

from the expression of the displacements

$$
\hat{\mathbf{u}}=\hat{\mathbf{u}}(x-w t, y, t), \quad \hat{\mathbf{u}}^{*}=\hat{\mathbf{u}}^{*}(x, y, t)
$$

the governing equations are: 
- for the top layer

$$
\left\{\begin{array}{l}
\left(1-\tau^{2} w^{2}\right) u_{, x x}+\tau^{2} u_{, y y}+\left(1-\tau^{2}\right) v_{, x y}=\tau^{2}\left(\ddot{u}-2 w \dot{u}_{, x}\right) \\
v_{, y y}+\tau^{2}\left(1-w^{2}\right) v_{, x x}+\left(1-\tau^{2}\right) u_{, x y}=\tau^{2}\left(\ddot{v}-2 w \dot{v}_{, x}\right)
\end{array}\right.
$$

- for the bottom layer

$$
\left\{\begin{array}{l}
u_{, x x}^{*}+\tau^{* 2} u_{, y y}^{*}+\left(1-\tau^{* 2}\right) v_{, x y}^{*}=\tau^{* 2} \ddot{u}^{*} \\
v_{, y y}^{*}+\tau^{* 2} v_{, x x}^{*}+\left(1-\tau^{* 2}\right) u_{, x y}^{*}=\tau^{* 2} \ddot{v}^{*}
\end{array}\right.
$$

Boundary and interface conditions are respectively:

$$
\begin{gathered}
u(x-w t, h, t)=0, \quad v(x, h, t)=\delta, u^{*}\left(x,-h^{*}, t\right)=v^{*}\left(x,-h^{*}, t\right)=0 \\
\sigma_{y y}(x, 0, t)=\sigma_{y y}^{*}(x, 0, t), \quad \sigma_{x y}(x, 0, t)=\sigma_{x y}^{*}(x, 0, t) .
\end{gathered}
$$

If there is contact, Coulomb's friction requires that:

$$
\sigma_{x y}^{*}(x, 0, t) \tilde{w}-f \sigma_{y y}^{*}(x, 0, t)|\tilde{w}|=0, \quad\left|\sigma_{x y}^{*}(x, 0, t)\right| \leq-f \sigma_{y y}^{*}(x, 0, t)
$$

where $\tilde{w}$ denotes the relative velocity

$$
\tilde{w}=\left(c_{2}^{*} \dot{u}^{*}-c_{2} w\left(u_{, x} / l+1\right)-c_{2} \dot{u}\right) .
$$

The steady sliding solution is:

$$
\left\{\begin{array}{l}
u(x-w t, y)=f(y-h) \frac{\rho^{*} c_{1}^{* 2} \delta}{\tau^{2}\left(\rho c^{2} h^{*}+\rho^{*} c^{* 2} h\right)} \\
v(x, y)=\delta+(y-h) \frac{\rho_{1}^{*} c_{1}^{*} \delta}{\left(\rho c_{1}^{2} h^{*}+\rho^{*} c_{1}^{* 2} h\right)} \\
u^{*}(x, y)=f\left(y+h^{*}\right) \frac{\rho c_{1}^{2} \delta}{\tau^{* 2}\left(\rho c_{1}^{2} h^{*}+\rho^{*} c^{* 2} h\right)} \\
v^{*}(x, y)=\left(y+h^{*}\right) \frac{\rho c_{1}^{2} \delta}{\left(\rho c_{1}^{2} h^{*}+\rho^{*} c_{1}^{* 2} h\right)} .
\end{array}\right.
$$

Stability analysis of the steady sliding solution The stability of the steady sliding solution can be obtained by a linearization of the dynamic equation under the assumption of sliding perturbed motions near the steady sliding state. These motions are searched for under the form

$$
\left\{\begin{array}{l}
\alpha=\frac{c}{c_{2}}, \quad \alpha^{*}=\frac{c}{c_{2}^{*}} \\
\mathbf{u}(x-w t, y, t)=e^{2 \pi \alpha t} e^{2 i k \pi(x-w t)} \mathbf{U}(y) \\
\mathbf{u}^{*}(x, y, t)=e^{2 \pi \alpha^{*} t} e^{2 i k \pi x} \mathbf{U}^{*}(y)
\end{array}\right.
$$

Thus $\mathbf{U}(y)$ and $\mathbf{U}^{*}(y)$ must satisfy

$$
\left\{\begin{array}{l}
-4 \pi^{2} k^{2}\left(1+\tau^{2}(\alpha-i w)^{2}\right) U+\tau^{2} U^{\prime \prime}+2 i k \pi\left(1-\tau^{2}\right) V^{\prime}=0 \\
-4 \pi^{2} k^{2}\left(\tau^{2}+\tau^{2}(\alpha-i w)^{2}\right) V+V^{\prime \prime}+2 i k \pi\left(1-\tau^{2}\right) U^{\prime}=0 \\
-4 \pi^{2} k^{2}\left(1+\tau^{* 2} \alpha^{* 2}\right) U^{*}+\tau^{* 2} U^{* \prime \prime}+2 i k \pi\left(1-\tau^{* 2}\right) V^{* \prime}=0 \\
-4 \pi^{2} k^{2}\left(\tau^{* 2}+\tau^{* 2} \alpha^{* 2}\right) V^{*}+V^{* \prime \prime}+2 i k \pi\left(1-\tau^{* 2}\right) U^{* \prime}=0
\end{array}\right.
$$


with

$$
\left\{\begin{array}{l}
U(h)=U^{*}\left(-h^{*}\right)=0 \\
V(0)=V^{*}(0) \\
\sigma_{x y}(0)=-f \sigma_{y y}(0) \Longleftrightarrow \tau^{2}\left(U^{\prime}(0)+2 i k \pi V(0)\right)=-f\left(V^{\prime}(0)+\left(1-2 \tau^{2}\right) 2 i k \pi U(0)\right) \\
\sigma_{x y}(0)=\sigma_{x y}^{*}(0) \Longleftrightarrow \rho c_{2}^{2}\left(U^{\prime}(0)+2 i k \pi V(0)\right)=\rho^{*} c_{2}^{* 2}\left(U^{*^{\prime}}(0)+2 i k \pi V^{*}(0)\right) \\
\sigma_{y y}(0)=\sigma_{y y}^{*}(0) \Longleftrightarrow \\
\rho c_{1}^{2}\left(V^{\prime}(0)+\left(1-2 \tau^{2}\right) 2 i k \pi U(0)\right)=\rho^{*} c_{1}^{* 2}\left(V^{*^{\prime}}(0)+\left(1-2 \tau^{2}\right) 2 i k \pi U^{*}(0)\right) .
\end{array}\right.
$$

It follows that

$$
\left\{\begin{array}{l}
U(y)=i p\left[P^{+} \cosh (2 k \pi p y)+P^{-} \sinh (2 k \pi p y)\right]+\left[Q^{+} \sinh (2 k \pi q y)+Q^{-} \cosh (2 k \pi q y)\right] \\
V(y)=\left[P^{+} \sinh (2 k \pi p y)+P^{-} \cosh (2 k \pi p y)\right]-i q\left[Q^{+} \cosh (2 k \pi q y)+Q^{-} \sinh (2 k \pi q y)\right] \\
\sigma_{x y}(y)=\frac{2 k \pi \rho c_{2}^{2}}{l}\left(\begin{array}{l}
\left(1+p^{2}\right) i\left(P^{+} \sinh (2 k \pi p y)+P^{-} \cosh (2 k \pi p y)\right) \\
+2 q\left(Q^{+} \cosh (2 k \pi q y)+Q^{-} \sinh (2 k \pi q y)\right)
\end{array}\right) \\
\sigma_{y y}(y)=\frac{2 k \pi \rho c_{2}^{2}}{l}\left(\begin{array}{l}
2 p\left(P^{+} \cosh (2 k \pi p y)+P^{-} \sinh (2 k \pi p y)\right) \\
-i\left(1+p^{2}\right)\left(Q^{+} \sinh (2 k \pi q y)+Q^{-} \cosh (2 k \pi q y)\right)
\end{array}\right)
\end{array}\right.
$$

where $(p, q)$ denotes any pair of complex numbers such that

$$
p^{2}=1+(\alpha-i w)^{2}, q^{2}=1+\tau^{2}(\alpha-i w)^{2} .
$$

The same expressions are obtained for $U^{*}(y), V^{*}(y)$ with $p^{* 2}=1+\alpha^{* 2}$ and $q^{* 2}=1+\tau^{* 2} \alpha^{* 2}$.

Boundary conditions lead to a system of linear equations for the unknowns $P^{+}, P^{-}, Q^{+}$, $Q^{-}, P^{*+}, P^{*-}, Q^{*+}, Q^{*-}$. The condition of existence of non null solutions requires that $c$ must be a root of the following equation:

$$
\begin{gathered}
\mathcal{F}(c)=\rho c_{2}^{2}(A(p, q, k h))\left(i B\left(p^{*}, q^{*}, k h^{*}\right)+f C\left(p^{*}, q^{*}, k h^{*}\right)\right)+ \\
\rho^{*} c_{2}^{* 2}\left(A\left(p^{*}, q^{*}, k h^{*}\right)\right)(i B(p, q, k h)-f C(p, q, k h))=0
\end{gathered}
$$

with

$p^{2}=1+\left(c / c_{2}-i w\right)^{2}, q^{2}=1+\tau^{2}\left(c / c_{2}-i w\right)^{2}, p^{* 2}=1+\left(c / c_{2}^{*}\right)^{2}, q^{* 2}=1+\left(\tau^{*}\right)^{2}\left(c / c_{2}^{*}\right)^{2}$

and

$$
\left\{\begin{aligned}
A(p, q, h)= & -4 p q\left(1+p^{2}\right)+p q\left(4+\left(1+p^{2}\right)^{2}\right) \cosh (2 \pi p k h) \cosh (2 \pi q k h) \\
& -\left(\left(1+p^{2}\right)^{2}+4 p^{2} q^{2}\right) \sinh (2 \pi p k h) \sinh (2 \pi q k h) \\
B(p, q, h)= & q\left(1-p^{2}\right)(\sinh (2 \pi p k h) \cosh (2 \pi q k h)-p q \cosh (2 \pi p k h) \sinh (2 \pi q k h)) \\
C(p, q, h)= & p q\left(3+p^{2}\right)-p q\left(3+p^{2}\right) \cosh (2 \pi p k h) \cosh (2 \pi q k h) \\
& +\left(2 p^{2} q^{2}+\left(1+p^{2}\right)\right) \sinh (2 \pi p k h) \sinh (2 \pi q k h) .
\end{aligned}\right.
$$

It is found that the existence of a root $c$ with positive real part is ensured when $f>0$. Thus the steady sliding solution is unstable.

\section{Particular cases}


The case of a rigid layer is obtained when $E \Rightarrow+\infty$ or $c_{2} \Rightarrow+\infty$. It follows that $c$ must satisfy the following equation

$$
\mathcal{F}(c)=i B\left(p^{*} q^{*}, k h^{*}\right)+f C\left(p^{*}, q^{*}, k h^{*}\right)=0
$$

when the top layer is rigid and

$$
\mathcal{F}(c)=i B(p, q, k h)-f C(p, q, k h)=0
$$

when the bottom layer is rigid. When the thickness $h \Rightarrow+\infty$, with the choice $\Re(p)<0$ and $\Re(q)<0$, the following expressions result:

$$
\begin{gathered}
A(p, q, \infty)=(1-p q)\left(\left(1+p^{2}\right)^{2}-4 p q\right), \quad B(p, q, \infty)=(1-p q) q\left(1-p^{2}\right) \\
C(p, q, \infty)=(1-p q)\left(\left(1+p^{2}\right)-2 p q\right) .
\end{gathered}
$$

For an elastic half-plane compressed into a moving rigid half-plane, cf. Martins et al. Martins et al. (1995), the results are:

$$
\mathcal{F}(c)=i q^{*}\left(1-p^{* 2}\right)+f\left(1+p^{* 2}-2 p^{*} q^{*}\right)=0 .
$$

In the case of two elastic half-planes, cf. Adams (1995), this equation can be written as:

$$
\begin{aligned}
& \mathcal{F}(c)=\rho c_{2}^{2}\left(\left(1+p^{2}\right)^{2}-4 p q\right)\left(i q^{*}\left(1-p^{* 2}\right)+f\left(1+p^{* 2}-2 p^{*} q^{*}\right)\right) \\
& +\rho^{*} c_{2}^{* 2}\left(\left(1+p^{* 2}\right)^{2}-4 p^{*} q^{*}\right)\left(i q\left(1-p^{2}\right)-f\left(1+p^{2}-2 p q\right)\right)=0 .
\end{aligned}
$$

The reader should refer to a more complete discussion given in this volume by Matins et al.

\section{On stick-slip waves}

It is well known that in the contact with friction of solids, a contact point may have a slip or stick or separation regime according to Coulomb's law of dry friction. For dynamical problems, the study of the propagation of these zones on the contact surface may be interesting in different applications (ultrasonic motors Zharii (1996), earthquake prediction and simulation Cochard and Madariaga (1995), mechanical vibrations and instabilities induced by friction Martins et al. (1995), Moirot (1998), Vola et al. (1999), Renard (1998), Nguyen (2000)). In the particular case of a solid in contact with a moving obstacle, the aforementioned results of the literature on the flutter instability of the steady sliding solution (Martins et al. (1995), Adams (1995)) suggested us to investigate for dynamic periodic responses in view of possible interpretation of this instability in connection with the notion of dynamic bifurcation. It is expected that a possible transition of the steady sliding response to a cyclic response in the spirit of Poincaré-Andronov-Hopf's bifurcation Nguyen (2000) may occur. For this reason, the search for periodic solutions is the first step to be investigated in the study of this transition. A simple system of equations with only one space variable is discussed here. It is derived from an academic modeling of a drum brake as a system of encased cylinders. The existence of a family of periodic solutions is established in this example under the form of stick-slip waves. Such an analytical solution is new in the literature. 


\subsection{An encased-cylinder problem and the reduced equations}

The mechanical response in plane strain of an elastic cylinder, of internal radius $R$ and external radius $R^{*}$, in frictional contact on its inner surface with a rotating rigid cylinder of radius $R+d$ and of angular rotation $\Omega$ as shown in Figure 8, is considered when the displacement is assumed to be homogeneous on its outer surface. Coulomb's law of dry friction is assumed with a constant friction coefficient $f$. An interesting approximation reducing this two-dimensional problem to an one-dimensional problem of contact with friction is discussed here. Our objective is to obtain explicitely the existence of periodic dynamic responses in the form of stick-slip waves. If stickslip solutions have been much discussed in static, we emphasize the fact that such a non-trivial example of stick-slip vibrations is not classically known in the literature for continuous systems. The construction of periodic dynamic responses have been principally discussed numerically Popp and Stelter (1990), Vola et al. (1999), Renard (1998), the works of Zharii (1996) on surface waves generated by piezo-electrical sources on a half-space boundary in contact with an obstacle remain an exception but his results deal only with slip waves. Non-dimensional variables are introduced

$$
\mathbf{u}=\frac{\overline{\mathbf{u}}}{R}, \quad \sigma=\frac{\bar{\sigma}}{E}, \quad r=\frac{\bar{r}}{R}, \quad \gamma=\frac{\rho R^{2} \Omega^{2}}{E}, \xi=\frac{R^{*}}{R}, \quad \delta=\frac{d}{R}, \quad t=\Omega \bar{t}, \dot{u}=\frac{d u}{d t}
$$

to write the governing equations in the form:

- kinematic relations $\epsilon=(\nabla \mathbf{u})_{s}$,

- fundamental law Div $\sigma=\gamma \ddot{\mathbf{u}}$,

- linear elasticity

$$
\sigma=\frac{\nu}{(1+\nu)(1-2 \nu)} \operatorname{Tr}(\epsilon) I+\frac{1}{1+\nu} \epsilon
$$

- boundary conditions

$$
u(\xi, \theta, t)=v(\xi, \theta, t)=0, \quad \sigma_{r r}(1, \theta, t)=-p(\theta, t), \quad \sigma_{r \theta}(1, \theta, t)=-q(\theta, t),
$$

- unilateral contact

$$
u \geq \delta, \quad p \geq 0, p(u-\delta)=0,
$$

- Coulomb's friction

$$
|q| \leq f p, \quad q(1-\dot{v})-f p|1-\dot{v}|=0 .
$$

The steady sliding solution is

$$
\left\{\begin{array}{c}
u_{e}=\delta \frac{1}{\xi^{2}-1}\left(\frac{\xi^{2}}{r}-r\right), v_{e}=\delta f \frac{1}{\xi^{2}-1}\left(\frac{\xi^{2}}{r}-r\right)\left(1+\frac{1}{\xi^{2}(1-2 \nu)}\right), \\
p_{e}=\delta \frac{1}{\xi^{2}-1} \frac{1}{1+\nu}\left(\xi^{2}+\frac{1}{1-2 \nu}\right)>0, q_{e}=f p_{e} .
\end{array}\right.
$$

An interesting modelling of the problem is obtained when the displacement is approximated in the form

$$
u=U(\theta, t) X(r), \quad v=V(\theta, t) X(r), \quad X(r)=\frac{1}{\xi^{2}-1}\left(\frac{\xi^{2}}{r}-r\right) .
$$


In this approximation, the following local equations are obtained from virtual work equation

$$
\left\{\begin{array}{l}
\ddot{U}-b U^{\prime \prime}-D V^{\prime}+g U=P, \\
\ddot{V}-a V^{\prime \prime}+D U^{\prime}+h V=Q, \\
P \geq 0, U-\delta \geq 0, \quad P(U-\delta)=0, \\
|Q| \leq f P, \quad Q(1-\dot{V})-f P|1-\dot{V}|=0 .
\end{array}\right.
$$

where ' denotes the derivative with respect to $\theta$ and

$$
\left\{\begin{array}{l}
a=\frac{\tilde{a} A}{\gamma B}, \quad b=\frac{\tilde{b} A}{\gamma B}, g=\frac{2 \tilde{a}+2\left(\xi^{2}-1\right) \tilde{b}}{\gamma B}, h=\frac{2 \xi^{2} \tilde{b}}{\gamma B}, \quad \tilde{a}=\frac{1-\nu}{(1+\nu)(1-2 \nu)}, \tilde{b}=\frac{1}{2(1+\nu)}, \\
A=-\frac{2 \xi^{2} \ln \xi}{\xi^{2}-1}+\frac{1+\xi^{2}}{2}>0, \quad B=\frac{\xi^{4} \ln \xi}{\xi^{2}-1}+\frac{1-3 \xi^{2}}{4}>0, \quad D=\frac{a C_{1}-b C_{2}}{A}, \\
C_{1}=\frac{2 \xi^{2} \ln \xi}{\xi^{2}-1}-1>0, C_{2}=-\frac{2 \xi^{2} \ln \xi}{\xi^{2}-1}-1+2 \xi^{2}>0 .
\end{array}\right.
$$

The coupling coefficient $D$ between the normal and tangential displacements can be positive or negative according to the values of $\nu$ and $\xi$. The steady sliding solution is given by $U_{e}=\delta$, $V_{e}=\delta f g / h, P=P_{e}$ and $Q_{e}=f P_{e}$.

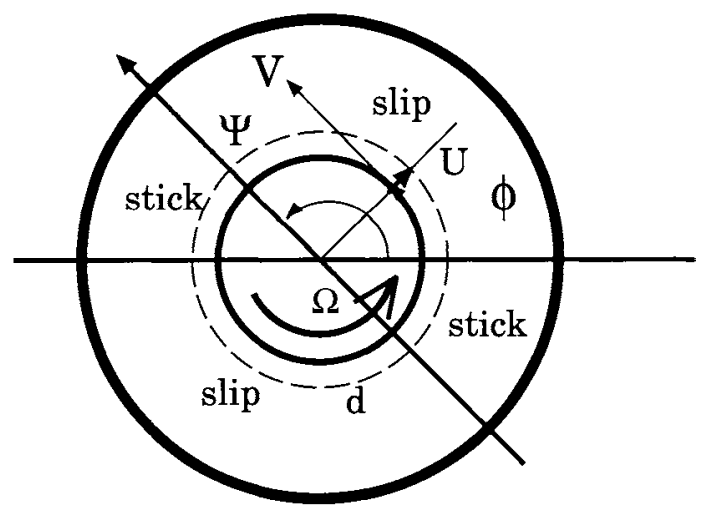

Figure 8: An example of encased cylinders. A stick-slip wave is represented in the case $k=2$.

\subsection{Instability of the steady sliding}

The stability of the steady sliding response is straightforward under the assumption of positive sliding motions. In this case a small perturbation motion is described by $U=U_{e}, V=V_{e}+V_{*}$, $P=P_{e}+P_{*}$ and $Q=Q_{e}+Q_{*}=f P$. It follows that

$$
\ddot{V}_{*}-a V_{*}^{\prime \prime}+f D V_{*}^{\prime}+h V_{*}=0 .
$$


A general solution is searched for under the form

$$
V_{*}=e^{s t} e^{i k \theta}
$$

It follows that

$$
-s^{2}=a k^{2}+h+i k f D .
$$

If $f=0$, then $s= \pm i \omega_{k}$ with $\omega_{k}=\sqrt{a k^{2}+h}$. Thus two harmonic waves propagating in opposite senses of the form $\cos \left(k \theta \pm \omega_{k} t+\varphi\right)$ are obtained as in classical elasticity. If $f>0$ and $D>0$, then

$$
s= \pm\left(s_{k}^{r}+i s_{k}^{i}\right), \quad s_{k}^{r}>0, \quad s_{k}^{i}<0
$$

thus a general solution of the form

$$
V_{*}=e^{ \pm s_{k}^{r} t} \cos \left(k \theta \pm s_{k}^{i} t+\varphi\right)
$$

is obtained and represents two waves propagating in opposite senses, an exploding wave in the sense of the implied rotation and a damping wave propagating in the opposite sense. If $f>0$ and $D<0$, the exploding wave propagates in the opposite sense since the previous expression of $s$ is still valid with $s_{k}^{r}>0$ and $s_{k}^{i}>0$. It is concluded again that the steady sliding response is unstable.

\subsection{Existence of stick-slip waves}

It is expected that in some particular situations, a dynamic bifurcation occurs in the spirit of Poincaré-Andronov-Hopf's bifurcation Nguyen (2000). This means that since the steady sliding response is unstable and there is flutter instability, the perturbed motion may eventually become a periodic response. This fact has been obtained numerically in many examples, cf. for example Vola et al. (1999), but a mathematical proof is still lacking since the classical proof of Hopf's theorem cannot be applied to the dynamic equations of solids in unilateral contact with dry friction.

The existence of possible periodic dynamic solutions is here our centre of interest. A periodic solution is searched for under the form of a wave propagating at constant velocity:

$$
U=U(\phi), \quad V=V(\phi), \phi=\theta-c t
$$

where $|c| R \Omega$ is the wave velocity, $U$ and $V$ are periodic functions of period $T=\frac{2 \pi}{k}$. The dynamic response is thus periodic of frequency $|c| k \Omega$. The propagation occurs in the sense of the rotation when $c>0$. According to the regime of contact, a slip wave or a stick-slip wave or a slip-separation wave or stick-slip-separation wave can be discussed. Only the two first cases are discussed here. The governing equations of such a wave follows from (72):

$$
\left\{\begin{array}{l}
\left(c^{2}-b\right) U^{\prime \prime}-D V^{\prime}+g U=P \\
\left(c^{2}-a\right) V^{\prime \prime}+D U^{\prime}+h V=Q \\
P \geq 0, U \geq \delta, \quad P(U-\delta)=0 \\
|Q| \leq f P, \quad Q(1-\dot{V})-f P|1-\dot{V}|=0
\end{array}\right.
$$


In the case of a slip wave, there is no separation and $Q=f P$ everywhere when there is positive slip $1-\dot{V} \geq 0$. Thus the governing equations of a positive slip wave are:

$$
U=\delta, \quad-D V^{\prime}+g \delta=P,\left(c^{2}-a\right) V^{\prime \prime}+h V=f P
$$

It follows that $V=V_{e}+\Delta$ with

$$
\left(c^{2}-a\right) \Delta^{\prime \prime}+f D \Delta^{\prime}+h \Delta=0
$$

Thus $\Delta=e^{r \phi}$ with

$$
r^{2}-2 \alpha r+\beta=0, \quad \alpha=\frac{-f D}{2\left(c^{2}-a\right)}, \beta=\frac{h}{c^{2}-a} .
$$

It follows that $r=\alpha \pm \sqrt{\alpha^{2}-\beta}$. This expression shows that no periodic solution can be obtained and thus no periodic response under the form of a positive slip wave exists.

A solution of (77) in the form of a stick-slip wave is now searched for. It is assumed that there is a positive slip regime in the interval $(0, \Psi T)$ and a stick regime in the interval $(\Psi T, T)$ : - in the stick region $(\Psi T, T)$, governing equations are

$$
U=\delta, \quad V^{\prime}=-\frac{1}{c}, P=\frac{D}{c}+g \delta>0, Q=h V,
$$

- in the slip region $(0, \Psi T)$ with positive slip, governimg equations are

$$
U=\delta, \quad P=g \delta-D V^{\prime}, \quad Q=f P, \quad\left(c^{2}-a\right) V^{\prime \prime}+f D V^{\prime}+h V-f g \delta=0 .
$$

The last equation shows that $V=V_{e}+\Delta$ in the interval $(0, \Psi T)$ with (79). The case $\alpha^{2}<\beta$ is considered thus $c^{2}>a+\frac{f^{2} D^{2}}{4 h}$. Let $\omega=\sqrt{\beta-\alpha^{2}}$. Since $r=\alpha \pm i \omega$, the general expression of $\Delta$ is

$$
\Delta=e^{\alpha \phi}(M \cos \omega \phi+N \sin \omega \phi) .
$$

There are 4 unknowns $(c, \Psi, M, N)$. The continuity of the displacement gives $V(0)=V(T)$. The stick region leads to two supplementary equations $V^{\prime}(0)=V^{\prime}(\Psi T)=-1 / c$. Thus, the following system of equations must be satisfied:

$$
\left\{\begin{array}{l}
M=e^{\alpha \Psi T}(M \cos \omega \Psi T+N \sin \omega \Psi T)-\frac{T(1-\Psi)}{c}, \\
\frac{-1}{c}=\alpha M+\omega N \\
\frac{-1}{c}=\alpha e^{\alpha \Psi T}(M \cos \omega \Psi T+N \sin \omega \Psi T)+\omega e^{\alpha \Psi T}(-M \sin \omega \Psi T+N \cos \omega \Psi T)
\end{array}\right.
$$

with restrictive conditions

$$
\left\{\begin{array}{l}
c^{2}>a+\frac{f^{2} D^{2}}{4 h} \\
|f g \delta+h \Delta|<f\left(\frac{D}{c}+g \delta\right), \quad 1+c V^{\prime} \geq 0 \quad \forall \phi \in(\Psi T, T) \\
g \delta-D \Delta^{\prime} \geq 0 \quad \forall \phi \in(0, \Psi T)
\end{array}\right.
$$

A family of stick-slip waves is considered for which the continuity of $Q$ at $\phi=T$ is ensured:

$$
Q\left(T_{-}\right)=Q\left(0_{+}\right) .
$$


This condition implies that

$$
h V\left(T_{-}\right)=f g \delta+\frac{f D}{c}
$$

and leads to the following system of governing equations and inequalities after the elimination of $M$ and $N$ :

$$
\left\{\begin{array}{l}
T(1-\Psi)+\frac{1}{\omega} e^{\alpha \Psi T} \sin \omega \Psi T=0 \\
1+\frac{\alpha}{\omega} e^{\alpha \Psi T} \sin \omega \Psi T-e^{\alpha \Psi T} \cos \omega \Psi T=0 \\
\alpha=\frac{-f D}{2\left(c^{2}-a\right)}, \quad \beta=\frac{h}{c^{2}-a}, \quad \omega=\sqrt{\beta-\alpha^{2}}, \quad c^{2}>a+\frac{f^{2} D^{2}}{4 h} \\
\left|f g \delta+h \Delta_{\Psi}\right| \leq \frac{f D}{c}+f g \delta, \quad 1+c \Delta^{\prime} \geq 0
\end{array}\right.
$$

The two first equations can be solved numerically by Mathematica and give $\Psi$ and $c$ for different values of $k$ and $f$. The variation of the stick ratio $1-\Psi$ as a function of $f$ is shown in Figure 9 . However, the condition of positive slip $1+c \Delta^{\prime} \geq 0$ can only be satisfied by some sets of data. For example, for $f=1$, only the cases $k=8,9,10,11,12$ lead to a positive slip solution. It is found that $c$ must have the sign of $D$ and the values of $c$ and $\Psi$ are independent of $\delta$ which must be strong enough. These waves propagate in the sense of the previous exploding perturbed motions with an amplitude independent on the applied displacement with a physical velocity independent on the rotation $\Omega$. For example, for $k=8, f=1$. the obtained results are $\Psi=0.839$ and $c=12.55$, the physical velocity is $\bar{c}=1255 \mathrm{~m} / \mathrm{s}$, cf. Figure 10 and Figure 11 .

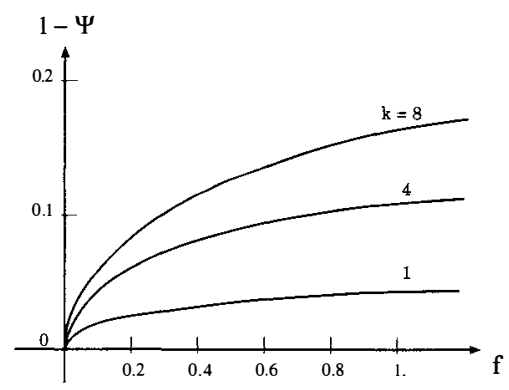

Figure 9: Dependence of the stick ratio on the friction coefficient. 


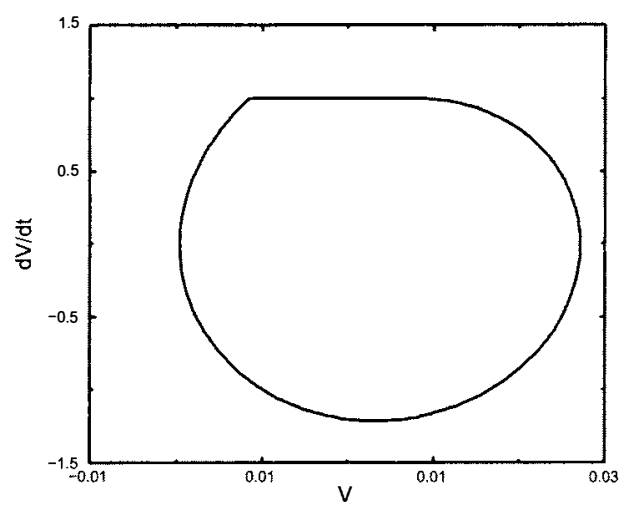

Figure 10: An example of stick-slip wave. The phase diagram of the periodic solution is given for $k=8, f=1$ and $\delta=0.005$.

\section{Remarks}

- In the presence of a stick-slip wave, the total pressure $\mathcal{P}$ is the same as in the steady gliding solution since

$$
\mathcal{P}=k \int_{0}^{T} P d \phi=\mathcal{P}_{e} .
$$

However as expected, the total moment $\mathcal{M}$ and the dissipation by friction $\mathcal{D}$ are lower than in the steady sliding solution since

$$
\begin{gathered}
\mathcal{M}=k \int_{0}^{T} Q d \phi=\mathcal{M}_{e}-\frac{k h T^{2}}{2|c|}(1-\Psi)^{2} \leq \mathcal{M}_{e} \\
\mathcal{D}=k \int_{0}^{T} Q\left(1+c V^{\prime}\right) d \phi=\mathcal{D}_{e}-\frac{k f D}{c}(1-\Psi) T-k f D c \int_{0}^{\Psi T} \Delta^{\prime 2} d \phi
\end{gathered}
$$

thus

$$
\mathcal{D}=\mathcal{M} \leq \mathcal{D}_{e}=\mathcal{M}_{e}
$$

- The next step to be explored is the growth of perturbed motions of the steady sliding solution. This is an open problem although some numerical simulations exist in the literature, cf. for example Oden and Martins (1985), Vola et al. (1999), Renard (1998). In particular, the formation of stick-slip waves can be found in Renard (1998) for the example of frictional sliding of a rectangular block on a rigid half-plane. 

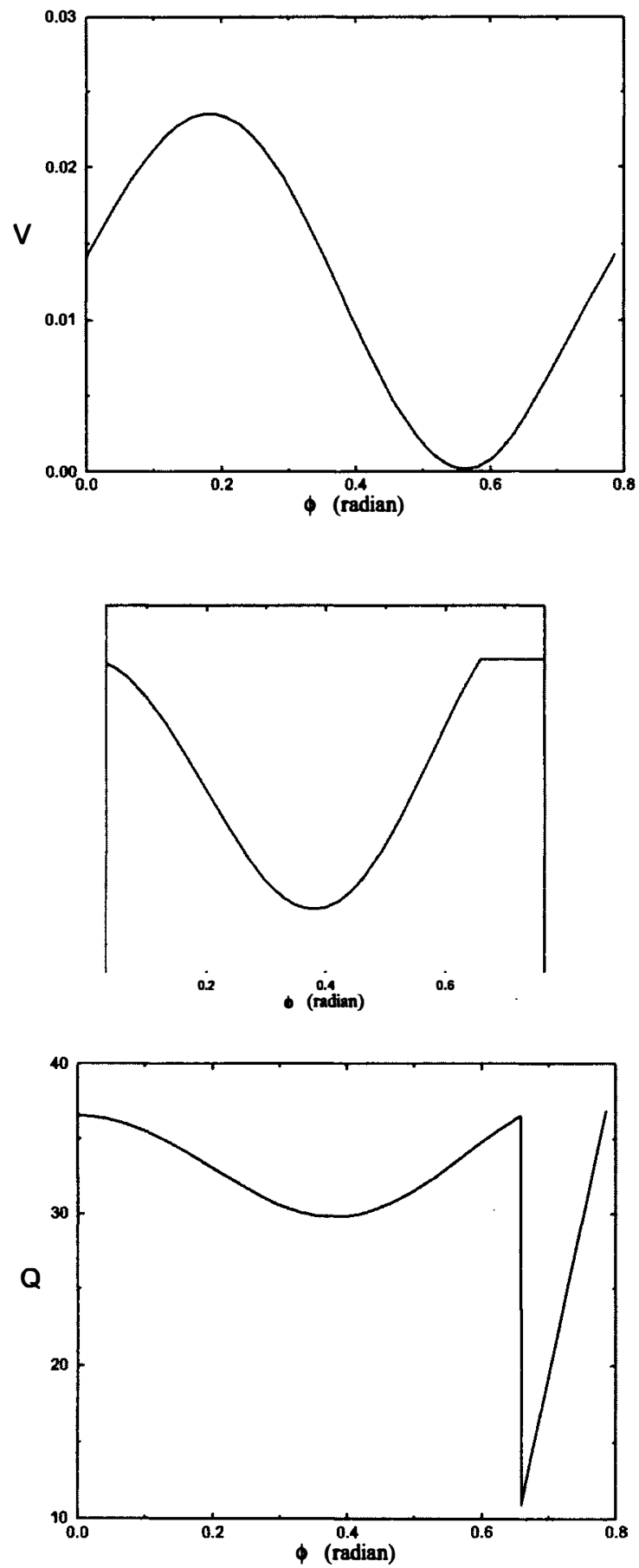

Figure 11: An example of stick-slip wave with $k=8, f=1$ and $\delta=0.005$. In this case, $\Psi=0.839, c=12.55$. The variations of $V, P$ and $Q$ are given as a function of $0 \leq \phi \leq 2 \pi / k$. 


\section{Application to the study of brake squeal}

The problem of brake noises has been intensively discussed in various experimental or theoretical investigations up to the present time. Different kinds of noises and vibrations can be identified in common drum or disk brakes following their frequencies. Brake squeals result from high frequency vibrations (greater than $5000 \mathrm{~Hz}$ ) and has a relatively pure spectrum composed of a few main frequencies accompanied by some harmonics. Although a brake squeal does not affect the mechanical behaviour of the brake, it is less and less accepted by the passengers. The conditions under which a squeal occurs are relatively well known. Most experiments showed that the brake squeal is excited more at low than at high speeds (less than $30 \mathrm{~km} / \mathrm{h}$ ). Squeal occurs only over limited ranges of brake pressure and is most prevalent at low temperature (less than $150^{\circ} \mathrm{C}$ ), cf. Chambrette and Jezequel (1992), Moirot (1998). The source of noise is attributed to the vibrations of brake components such as drum or pad and disk and brake noises are generated mainly by the sliding phenomena.

Our objective is to present in this paper a mechanical analysis of brake squeal. The principal interpretation is the fact that brake squeal is a consequence of the flutter instability of the steady sliding solution of the pad on the disk for a disk brake. This analysis gives the system of governing equations to be considered and enables us to determine the steady sliding solution. The fact that the disk is in rotation can be easily taken into account since the system remains in small strains. The determination of the steady sliding solution is discussed in function of the coefficient of friction. In particular, the conditions ensuring the existence or the uniqueness of the steady sliding solution are discussed since for high friction, the steady sliding solution may be not unique or does not even exist.

The stability of a steady sliding solution is considered in a second part. Perturbed motions of the mechanical system of pad and disk near the steady sliding solution are introduced. Under the assumption of a slip regime, the dynamic solution can be simplified and the perturbed slip motions can be considered. Stability analysis with respect to these motions can be discussed and leads to the resolution of an generalized eigenvalue problem with unsymmetric mass and rigidity matrices. This non-symmetry is due to the presence of friction as well as of the disk rotation. The existence of an eigenvalue with a positive real part and a non-zero imaginary part implies necessarily an instability by flutter of the steady sliding solution.

The numerical aspect of the problem is considered in a third part. The steady sliding solution can be determined by a resolution of the nonlinear problem in the vehicle reference by iterations. Once this solution obtained, its stability analysis can be performed. The adopted procedure is based upon a modal basis of free vibrations. The generalized eigenvalue problem is solved in this basis of functions and the eigenvectors are generated by a finite number of first vibration modes. The numerical procedure is first validated on the problem of contact with friction of infinite elastic layers which has been discussed analytically by Adams (1995) and by Martins et al. (1995). Then an example of disk brake is considered.

\subsection{Governing equations}

The system of disk and pad is considered in the vehicle axes $(O x)$, which is a Galilean reference since the vehicle motion is assumed to be at constant velocity. In this reference, the pad is an elastic solid $P$ in small deformation. For simplicity, it is assumed that this solid is under the 


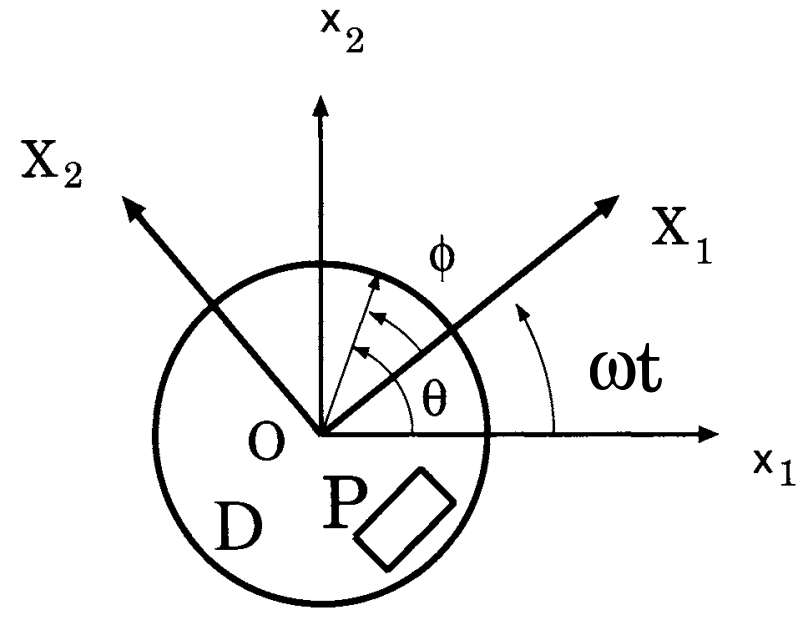

Figure 12: A model of disk brake

action of implied displacements (by the brake action) and contact forces with the disk on a potential contact surface $S$. If $u^{P}(x, t)$ and $\sigma^{P}(x, t)$ denote respectively the displacement and stress at the point $x$ and time $t$, the dynamic equations for the pad $P$ are

$$
\sigma^{P}=L: \nabla u^{P}, \quad \text { Div } \sigma^{P}-\rho u^{P}{ }_{t t}=0 \forall x \in P, \sigma^{P} \cdot n^{P}=R \forall x \in S .
$$

In this expression, $R$ is the reaction of the disk $D$, which is related to the relative displacement and velocity of the two solids following the unilateral contact and Coulomb's law of dry friction with a constant coefficient of friction.

Let $\omega$ be the rotation velocity of the disk around the axis $O x_{3}$. It is convenient to consider the rotating axes $(O X)$, where $O X_{3}$ coincides with $O x_{3}$. The equations of motion of the solid $D$ in axes $(O X)$ are simply the dynamic equations of an elastic solid in small deformation in a relative reference

$$
\Sigma=L: \nabla U, \quad \operatorname{Div} \Sigma-\rho \gamma=0, \forall X \in D_{o}
$$

with

$$
\gamma=\gamma_{e}+\gamma_{r}+2 \omega \mathbf{k} \wedge V_{r}=-\omega^{2} \mathbf{r}+U_{, t}+2 \omega \mathbf{k} \wedge U_{t}
$$

where $D_{o}$ denotes the undeformed volume of the disk in these axes and $\Sigma=\Sigma(X, t), \quad U=$ $U(X, t)$ are respectively the stress tensor and displacement vector. It is necessary to adopt the vehicle axes $(O x)$ and a change of variable must be introduced. This change of variable can be expressed in terms of the cylindrical coordinates as shown in Figure 12:

$$
X=(r, \phi, z), \quad x=(r, \theta, z), \theta=\phi+\omega t .
$$

The change of variable

$$
\begin{aligned}
u(r, \theta, z, t) & =U(r, \theta-\omega t, z, t), \quad U(r, \phi, z, t)=u(r, \phi+\omega t, z, t) \\
\sigma(r, \theta, z, t) & =\Sigma(r, \theta-\omega t, z, t), \quad \Sigma(r, \phi, z, t)=\sigma(r, \phi+\omega t, z, t)
\end{aligned}
$$


gives in the Galilean axes $(O x)$

$$
\begin{gathered}
\sigma=L: \nabla u, \quad \text { Div } \sigma-\rho \gamma=0, \forall x \in D, \\
\gamma=\omega^{2}(-\mathbf{r}+u, \theta \theta+2 \mathbf{k} \wedge u, \theta)+2 \omega(u, \theta t+\mathbf{k} \wedge u, t)+u, t t
\end{gathered}
$$

with the boundary condition

$$
\sigma \cdot n=-R, \forall x \in S
$$

and implied displacements along the axis of rotation $O x_{3}$. It is also important to give the expression of the material velocity $v(x, t)$ in this reference. From the expression of the velocity of a material point in the rotating axes

$$
v=V_{e}+V_{r}=\omega \mathbf{k} \wedge \mathbf{r}+U_{t}
$$

it follows that

$$
v(x, t)=\omega(\mathbf{k} \wedge \mathbf{r}+u, \theta)+u, t .
$$

The conditions of unilateral contact and dry friction of the solids $P$ and $D$ can be written now. On the surface $S$ of contact, which is assumed initially without normal gap, the condition of non-penetration is

$$
[u]_{N}=\left(u^{P}-u\right) \cdot n \geq 0, \quad \forall x \in S .
$$

The unilateral contact condition gives

$$
[u]_{N} \geq 0, \quad R_{N} \geq 0,[u]_{N} R_{N}=0 \forall x \in S .
$$

The relative velocity of material points in contact is

$$
w(x, t)=u,_{t}^{P}(x, t)-v(x, t)=[u]_{,}-\omega \mathbf{k} \wedge \mathbf{r}-\omega u,{ }_{\theta} .
$$

Coulomb's friction law can be written as

$$
w=\nu R_{T}, \quad \varphi=\left\|R_{T}\right\|-f R_{N} \leq 0, \nu \leq 0, \nu \varphi=0 .
$$

The previous expressions of $\gamma$ and of $w$ show that a possible approximation of the solution is obtained by assuming that the relative velocity is due simply to the rotation of the disk and one can retain in the dynamic equation of the disk only the centrifuge forces and neglect all other terms of rotation. This approximation $\mathcal{A}$ consists of writing

$$
\gamma=-\omega^{2} \mathbf{r}+u, t t, w(x, t)=[u]_{, t}-\omega \mathbf{k} \wedge \mathbf{r} .
$$

\subsection{Steady sliding solution}

The steady state solution is obtained when $u(x, t)$ does not depend on $t$. Thus, the steady state solution is governed by the following equations

$$
\left\{\begin{array}{l}
\sigma^{P}=L: \nabla u^{P}, \quad \text { Div } \sigma^{P}=0, \quad \forall x \in P, \sigma^{P} \cdot n=-R, \quad \forall x \in S \\
\sigma=L: \nabla u, \quad \text { Div } \sigma-\rho \gamma=0, \quad \forall x \in D, \quad \sigma \cdot n=-R, \quad \forall x \in S \\
\gamma=\omega^{2}(-\mathbf{r}+u, \theta \theta+2 \mathbf{k} \wedge u, \theta)
\end{array}\right.
$$


together with the equations (97), (98), (100) and the following expression of the relative velocity

$$
w(x, t)=-\omega\left(\mathbf{k} \wedge \mathbf{r}+u,_{\theta}\right) .
$$

The approximation $\mathcal{A}$ consists of solving the following equations

$$
\left\{\begin{array}{l}
\sigma^{P}=L: \nabla u^{P}, \quad \text { Div } \sigma^{P}=0, \quad \forall x \in P, \sigma^{P} \cdot n=-R, \forall x \in S, \\
\sigma=L: \nabla u, \quad \operatorname{Div} \sigma+\rho \omega^{2} \mathbf{r}=0, \quad \forall x \in D, \sigma \cdot n=-R, \forall x \in S
\end{array}\right.
$$

together with equations (97), (98), (100) with $w(x, t)=-\omega \mathbf{k} \wedge \mathbf{r}$. Within this approximation, since the relative velocity is not zero, the transverse reaction $R_{T}$ has the direction of $-\tau$ with $\tau=\frac{w}{\|w\|}$. Finally, the steady sliding solution is given by the equations (97), (98) and

$$
\left\{\begin{array}{l}
\sigma^{P}=L: \nabla u^{P}, \quad \text { Div } \sigma^{P}=0 \forall x \in P, \sigma^{P} \cdot n=-R_{N} n+f R_{N} \tau, \forall x \in S, \\
\sigma=L: \nabla u, \quad \text { Div } \sigma+\rho \omega^{2} \mathbf{r}=0, \forall x \in D, \sigma \cdot n=-R_{N} n+f R_{N} \tau, \forall x \in S .
\end{array}\right.
$$

These equations can also be written in the form

$$
\begin{gathered}
\int_{P} \nabla u^{P}: L: \nabla \delta u d V+\int_{D} \nabla u: L: \nabla \delta u d V-\int_{D} \omega^{2} r \cdot \delta u d V+ \\
\int_{S}\left(R_{N}[\delta u]_{N}+f R_{N} \tau \cdot[\delta u]_{T}\right) d S=0,
\end{gathered}
$$

together with (97) and (98). To understand the mathematical nature of the problem of steady sliding in the approximation $\mathcal{A}$, let us consider the associated discrete problem obtained from these equations after a discretization by the finite element method for example. If $U=\left(U_{N}, U_{T}, U_{Z}\right)$ denotes for each solid the degrees of freedom representing respectively the normal, tangential displacements of the contact surface and other complementary displacements in the solid, the governing equations are, for each solid

$$
\left[\begin{array}{lll}
K_{N N} & K_{N T} & K_{N Z} \\
K_{T N} & K_{T T} & K_{T Z} \\
K_{Z N} & K_{Z T} & K_{Z Z}
\end{array}\right]\left[\begin{array}{c}
U_{N} \\
U_{T} \\
U_{Z}
\end{array}\right]=\left[\begin{array}{c}
R_{N} \\
f[\Phi] R_{N} \\
F_{Z}
\end{array}\right]
$$

where $\Phi$ denotes an appropriated matrix. The elimination of $U_{Z}$ leads to a matrix equation in terms of $\Delta=U^{P}-U$

$$
\left[\begin{array}{cc}
k_{N N} & k_{N T} \\
k_{T N} & k_{T T}
\end{array}\right]\left[\begin{array}{c}
\Delta_{N} \\
\Delta_{T}
\end{array}\right]=\left[\begin{array}{c}
R_{N}+F_{N} \\
f[\Phi] R_{N}+F_{T}
\end{array}\right]
$$

Finally, the normal displacement $\Delta_{N}$ is related to the normal reaction $R_{N}$ by

$$
\begin{gathered}
{\left[k_{N N}-k_{N T} k_{T T}^{-1} k_{T N}\right]\left[\Delta_{N}\right]=\left[I-f k_{N T} k_{T T}^{-1} \Phi\right]\left[R_{N}\right]+[g]} \\
\Delta_{N}^{k} \geq 0, \quad R_{N}^{k} \geq 0, \quad \Delta_{N}^{k} R_{N}^{k}=0, \quad \forall k=1, m .
\end{gathered}
$$

This is a linear complementarity problem, cf. Cottle et al. (1992), Isac (1992), Klarbring (1986), Klarbring (1997), Nguyen (2000):

$$
\left[\Delta_{N}\right]=[A]\left[R_{N}\right]+[F], \quad \Delta_{N}^{k} \geq 0, \quad R_{N}^{k} \geq 0, \quad \Delta_{N}^{k} R_{N}^{k}=0 .
$$


with

$$
[A]=\left[k_{N N}-k_{N T} k_{T T}^{-1} k_{T N}\right]^{-1}-f\left[k_{N N}-k_{N T} k_{T T}^{-1} k_{T N}\right]^{-1}\left[k_{N T} k_{T T}^{-1} \Phi\right] .
$$

It is recalled that for a given $[F]$, this problem has one and only one solution if the matrix $[A]$ is a P-matrix. This property means that $[A]$ satisfies the condition of P-positivity

$$
[A] \text { is a P-matrix } \Leftrightarrow \exists i \text { such that } \sum_{j} X_{i} A_{i j} X_{j}>0 \quad \forall X \neq 0 .
$$

In fact, the existence of a solution is still ensured if $[A]$ satisfies only a co-P-positivity condition:

$$
[A] \text { is a co-P-matrix } \Leftrightarrow \exists i \text { such that } \sum_{j} X_{i} A_{i j} X_{j}>0 \quad \forall X \neq 0, \quad X \geq 0 .
$$

This condition is similar to the P-positivity condition but is concerned only with vectors of positive components. It has been established for a matrix not necessarily symmetric that

$$
\begin{array}{ccc}
\text { Positive-definiteness } & \Rightarrow \text { P-positivity } \Rightarrow \text { Co-P-positivity, } \\
\text { Positive-definiteness } \Rightarrow \text { Co-positivity } \Rightarrow \text { Co-P-positivity. }
\end{array}
$$

For symmetric matrices, it is also true that P-positivity and positive-definiteness are equivalent

$$
\text { Positive-definiteness } \Leftrightarrow \text { P-positivity. }
$$

When the full expressions (102), (103) of $\gamma$ and $w$ are taken into account, the discussion can be done in the same spirit and leads again to a complementarity problem.

The significance of the quadratic form $\left[R_{N}\right]^{T}[A]\left[R_{N}\right]$ is very simple. It represents the work done by the normal reactions $R_{N} \Delta_{N}$ of the system when when submitted to the contact reaction $R=R_{N} n+f R_{N} \tau$ and to homogeneous implied displacement. The condition of positivity states that for any non-zero distribution of normal reactions, the work done by this distribution is positive. The condition of co-P-positivity is much weaker, it states that for any non-zero and positive distribution of the normal reactions, there exists at least one strictly positive normal displacement associated with a strictly positive normal reaction.

The contribution of the rotation terms to the rigidity matrix of the solid $D$ corresponds to some additional symmetric matrices since the following expressions hold

$$
\begin{gathered}
\int_{D} u,_{\theta \theta} \cdot \delta u d V=-\int_{D} u,_{\theta} \delta u,,_{\theta} d V \\
\int_{D}\left(\mathbf{k} \wedge u,_{\theta}\right) \cdot \delta u d V=\int_{D} \operatorname{det}[k, u, \theta, \delta u] d V= \\
=\int_{D}-\operatorname{det}\left[k, u, \delta u,{ }_{\theta}\right] d V=\int_{D} \operatorname{det}[k, \delta u, \theta, u] d V .
\end{gathered}
$$

After a partial integration with respect to $\theta$ on the interval $[0,2 \pi]$, these expressions are symmetric with respect to $(u, \delta u)$. Under the assumption of small rotations, $\omega$ remains sufficiently small, the contribution of these additional terms to the rigidity of solid $D$ does not change its positivity.

The fact that the condition co-P-positivity is satisfied depends on the considered problem. Even for elastic solids under small deformations, it is not difficult to give simple examples showing that the possibility of steady sliding does not exist for a sufficiently high friction coefficient. 


\subsection{Brake squeal: a problem of flutter instability of the steady sliding response?}

The stability of the steady sliding solution is obtained from the behaviour of the perturbed motions of the system near the considered steady solution. This discussion is a priori difficult since a small perturbed motion near the steady solution is not necessarily governed by linear equations. It is well known that the unilateral contact and Coulomb's laws are non-smooth and cannot be linearized at the steady state. It is assumed first that the steady sliding solution satisfies on the contact surface $S$ the condition of effective contact

$$
R_{N}(x)>0 \quad \forall x \in S .
$$

It is then expected that a small perturbed motion cannot lead to a separation of contact at any point of $S$, at least at the early time. This remark enables us to consider only in-contact motions of the solids on contact surface $S$ to avoid the difficulties related to the unilateral aspect.

Even in these motions, it is necessary to separate the slip regime $w \neq 0$ from the stick regime $w=0$. The problem of stick-slip motions has been much discussed in the literature. In particular, for a simple oscillator, the modification of the initial frequency has been considered, cf. for example Popp and Stelter (1990) for a velocity dependent coefficient of friction. The stickslip motion has been computed for a three-dimensional oscillator by Cho and Barber (1999). The stick-slip motions play an important role in most contact problems cf. Zharii (1996), in particular in the study of noise emission. However, the presence of stick-slip motions is a source of difficulty and in this stability analysis, only the slip motions will be explored. Under this restriction, the equations of motion can be effectively linearized near the steady sliding solution. Let $u^{*}$ and $\sigma^{*}$ are the difference

$$
u^{*}=u_{p}-u_{e}, \quad \sigma^{*}=\sigma_{p}-\sigma_{e}
$$

where $u_{e}, \sigma_{e}$ refer to the steady sliding solution and $u_{p}, \sigma_{p}$ to the perturbed motion. For small perturbations, $u^{*}$ and $\sigma^{*}$ are governed by the linearized equations at the steady sliding state. Thus, the following equations hold for the slip motions in the vicinity of the steady sliding state:

$$
\left\{\begin{array}{l}
\sigma^{* P}=L: \nabla u^{* P}, \quad \operatorname{Div} \sigma^{* P}-\rho u^{* P}, t t=0 \forall x \in P, \sigma^{* P} \cdot n=-R^{*}, \forall x \in S, \\
\sigma^{*}=L: \nabla u^{*}, \quad \operatorname{Div} \sigma^{*}-\rho \gamma^{*}=0, \quad \forall x \in D, \sigma^{*} \cdot n=-R^{*}, \forall x \in S, \\
\gamma^{*}=\omega^{2}\left(u^{*}, \theta \theta+2 \mathbf{k} \wedge u^{*},_{\theta}\right)+2 \omega\left(u^{*}, \theta t+\mathbf{k} \wedge u^{*}, t\right)+u^{*}, t t,\left[u^{*}\right]_{N}=0 .
\end{array}\right.
$$

In these equations, the tangent reaction $R_{T}^{*}$ is related to the normal reaction $R_{N}^{*}$ by the linearized expression of the equation

$$
R_{T}=-f R_{N} \frac{w}{\|w\|}
$$

which gives

$$
\begin{gathered}
R_{T}^{*}=-f R_{N}^{*} \tau-f R_{N}\left(\frac{w^{*}}{\|w\|}-w \frac{w \cdot w^{*}}{\|w\|^{3}}\right) \text { with } \\
w^{*}=\left[u^{*}, t\right]-\omega u^{*}, \theta .
\end{gathered}
$$

These equations can also be conveniently written under the variational form of the virtual work equation. For the solid $P$, the classical equation

$$
\int_{P} \rho u^{* P}, t t \cdot \delta u d V+\int_{P} \nabla u^{* P}: L: \nabla \delta u d V=\int_{S} R^{*} \cdot \delta u d S
$$


is obtained. For the solid $D$, the following equation holds

$$
\begin{gathered}
\int_{D} \rho u^{*}, t t \cdot \delta u d V+2 \omega \int_{D} \rho\left(u^{*}, \theta t \cdot \delta u+\operatorname{det}\left[k, u^{*}, t, \delta u\right]\right) d V \\
+\int_{D} \nabla u^{*}: L: \nabla \delta u d V+\omega^{2} \int_{D}\left(u^{*},{ }_{\theta \theta} \cdot \delta u+2 \operatorname{det}\left[k, u^{*},{ }_{\theta}, \delta u\right]\right) d V=-\int_{S} R^{*} \cdot \delta u d S .
\end{gathered}
$$

In this equation, it should be noted that the terms involving $u^{*}{ }_{t}$ are skew-symmetric while the terms involving $u^{*},{ }_{t t}$ or $u$ are symmetric. If the solution is searched for in the form $\left(u^{*}, R^{*}\right)=$ $(d(x), r(x))$ exp st, the eigenvalues $s$ and eigenvectors $d$ must satisfy the equation:

$$
s^{2} \int_{P} \rho d^{P} \cdot \delta u d V+\int_{P} \nabla d^{P}: L: \nabla \delta u d V=\int_{S} r \cdot \delta u d S
$$

for the solid $P$. For the solid $D$, the following equation holds

$$
\begin{gathered}
s^{2} \int_{D} \rho d^{D} \cdot \delta u d V+s 2 \omega \int_{D} \rho\left(d^{D},{ }_{\theta} \cdot \delta u+\operatorname{det}\left[k, d^{D}, \delta u\right]\right) d V+ \\
+\int_{D} \nabla d^{D}: L: \nabla \delta u d V+\omega^{2} \int_{D}\left(-d^{D},{ }_{\theta} \cdot \delta u,{ }_{\theta}+2 \operatorname{det}\left[k, d^{D},_{\theta}, \delta u\right]\right) d V=-\int_{S} r \cdot \delta u d S .
\end{gathered}
$$

After a discretization by the finite element method with the nodal shape functions $\mathcal{N}_{i}(x)$

$$
d(x)=\sum_{i=1}^{n} d_{i} \mathcal{N}_{i}(x)
$$

the following matrix equations hold:

$$
\left(s^{2}\left[M^{P}\right]+\left[K^{P}\right]\right)\left[d^{P}\right]=\left[r^{P}\right], \quad\left(s^{2}\left[M^{D}\right]+s\left[G^{R}\right]+\left[K^{D}\right]+\left[K^{D R}\right]\right)\left[d^{D}\right]=-\left[r^{D}\right]
$$

with $\left[d^{P}\right]=\left[d_{P}, d_{P T}, d_{N}\right]^{T},\left[d^{D}\right]=\left[d_{N}, d_{D T}, d_{D}\right]^{T}$. where the notation $d_{N}, d_{D T}, d_{D}$ refers to different nodal values of solid $D$, respectively to the normal and tangent nodal displacements on the contact surface and to other nodal displacements elsewhere. Note that $\left[G^{R}\right]$ is skewsymmetric and $\left[K^{R}\right]$ is symmetric matrix. Thus, the effect of the rotation of $D$ is finally expressed by a gyroscopic term and by an additional symmetric rigidity. The force matrices $\left[r^{P}\right]$ and $\left[r^{D}\right]$ are related by

$$
\left[r^{P}\right]=\left[0, r_{T}, r_{N}\right]^{T}, \quad\left[r^{D}\right]=\left[r_{N}, r_{T}, 0\right]^{T} .
$$

From the expression of $R_{T}^{*}$, the following equation holds

$$
\left[r_{T}\right]=f[\Phi]\left[r_{N}\right]+f s[A]\left[d_{P T}-d_{D T}\right]+f\left[B_{T}\right]\left[d_{D T}\right]+f\left[B_{N}\right]\left[d_{N}\right]
$$

where $[A],\left[B_{T}\right],\left[B_{N}\right]$ are some appropriated square matrices. From the expression of $\left[r_{N}\right]$

$$
\left[r_{N}\right]=\left(s^{2}\left[M_{N .}^{P}\right]+\left[K_{N .}^{P}\right]\right)\left[d^{P}\right]=-\left(s^{2}\left[M_{N .}^{D}\right]+s\left[G_{N .}^{R}\right]+\left[K_{N .}^{D}\right]+\left[K_{N .}^{R}\right]\right)\left[d^{D}\right],
$$

$r_{T}$ and $r_{N}$ can be written in terms of the displacement $[d]=\left[d_{P}, d_{P T}, d_{N}, d_{D T}, d_{D}\right]^{T}$. Finally, the eigenvalues $s$ and eigenvectors $[d]$ must satisfy

$$
\left\{s^{2}([M]-f[m])+s([G]-f[\ell])+[K]+\left[K^{R}\right]-f[k]\right\}[d]=[0] \text { with }
$$




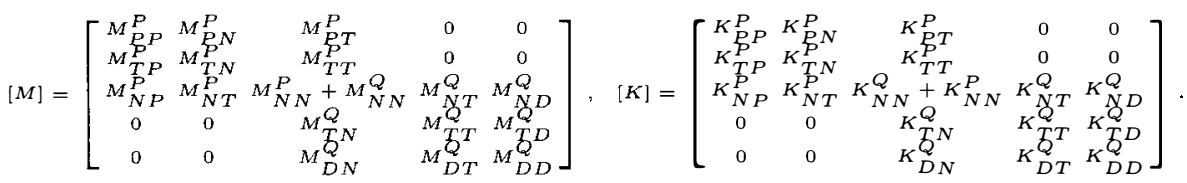

$$
\begin{aligned}
& {[m]=\left[\begin{array}{ccccc}
0 & 0 & 0 & 0 & 0 \\
\Phi M_{N P}^{P} & \Phi M_{N T}^{P} & \Phi M_{N N}^{P} & 0 & 0 \\
0 & 0 & 0 & 0 \\
0 & 0 & -\Phi M_{N N}^{D} & -\Phi M_{N T}^{D} & -\Phi M_{N D}^{D} \\
0 & 0 & 0 & 0 & 0
\end{array}\right], \quad[\ell]=\left[\begin{array}{ccccc}
0 & 0 & 0 & 0 & 0 \\
0 & A & 0 & -A & 0 \\
0 & 0 & 0 & 0 \\
0 & -A & -\Phi G_{N N}^{D R} & A-\Phi G_{N T}^{D R} & 0 \\
0 & 0 & 0 & 0 & 0 \\
& & & 0 & 0
\end{array}\right],} \\
& {[k]=\left[\begin{array}{ccccc}
0 & 0 & 0 & 0 & 0 \\
\Phi K_{N P}^{P} & \Phi K_{N T}^{P} & \Phi K_{N N}^{P}+B_{N} & 0 \\
0 & 0 & B_{T} & 0 \\
0 & 0 & -\Phi\left(K^{D}+K^{D R}\right)_{N N}-B_{N} & -\Phi\left(K^{D}+K^{D R}\right)_{N T}-B_{T}-\Phi\left(K^{D}+K^{D R}\right)_{N D} \\
0 & 0 & 0 & 0
\end{array}\right] \text {, }}
\end{aligned}
$$

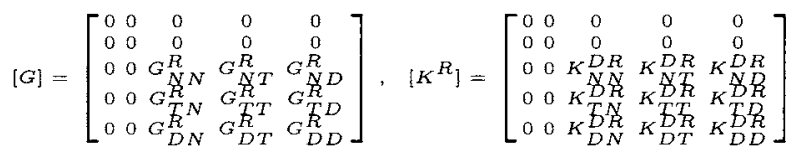

This eigenvalue problem can be written as

$$
\begin{gathered}
\left(s^{2}[\bar{M}]+s[\bar{Z}]+[\bar{K}]\right)[d]=0 \\
{[\bar{M}]=[M]-f[m], \quad[\bar{Z}]=[G]-f[\ell], \quad[\bar{K}]=[K]+\left[K^{R}\right]-f[k] .}
\end{gathered}
$$

Thus the presence of friction breaks the symmetry of the mass and rigidity matrices in the eigenvalue problem to be solved. Since the numerical resolution of this non-symmetric problem of dimension $n$ is time-consuming, a reduction of the dimension of the problem is necessary. For this, it is interesting to consider the basis of vibration modes $D_{k}$ of the perfect associated system defined by the equations (of dimension $n$ )

$$
\left(s_{o}^{2}[M]+[K]\right)[D]=[0] .
$$

Let $D_{k}, k=1, m$ denote the first $m$ vibration modes. The reduction of variable consists to search for $[d]$ in this basis:

$$
[d]=\left[\begin{array}{llll}
D_{1} & D_{2} & \ldots & D_{m}
\end{array}\right]\left[q_{1} \quad \ldots q_{m}\right]^{T}=[C][q] .
$$

Finally, the eigenvalue $s$ and eigenvector $[q]$ are defined by an eigenvalue problem of dimension $m$

$$
\begin{gathered}
\left(s^{2}[\mathcal{M}]+s[\mathcal{Z}]+[\mathcal{K}]\right)[q]=[0] \\
{[\mathcal{M}]=[C]^{T}[\bar{M}][C], \quad[\mathcal{Z}]=[C]^{T}[\bar{Z}][C], \quad[\mathcal{K}]=[C]^{T}[\bar{K}][C]}
\end{gathered}
$$

The resolution of (133) leads to the complex eigenvalues $s_{k}$ and complex eigenvectors $Q_{k}$. Since $u^{*}(x, t)=\mathcal{N}_{i}(x) \Re\left(\sum_{k} a_{k} C_{i j} Q_{k j} \exp s_{k} t\right)$, it is concluded that the steady sliding solution is unstable if there exists an eigenvalue with a positive real part. Although not necessary, the choice of this modal basis is interesting since the dynamic behaviour of the system is well generated by a small number of modes, in practice $m \ll n$. 


\subsection{Validation}

The proposed numerical procedure is first validated on a sample problem which is the sliding of a rigid plate on an elastic infinite layer.

The modal basis $D_{k}, k=1, m$ is first computed by the resolution of the equations (131). As usual, these frequencies of vibration can be obtained with great precision. The relative error of the computed frequencies as a function of the mesh size is less than $1 \%$ for the 30 first frequencies with the mesh $12 \times 36$. Figure 5.5 presents some modes and the associated frequencies of vibration.

The resolution of the equation (133) for a chosen basis $D_{k}, k=1, m$ can be done following the standard methods available for example in the code Nastran. Hessenberg's method seems to give good numerical results. It is recalled that Hessenberg's method is obtained in two steps, a reduction to a Hessenberg matrix and an iteration by a QR algorithm. This method gives all eigenvalues and the associated eigenvectors are obtained by inverse iterations.

The variations of the frequencies as a function of the friction coefficient are obtained numerically with less than $3 \%$ error compared to the exact values. It is found that good results can be obtained with a small number of modes, in practice $m=200$ is sufficient. The numerical results show that the steady sliding solution is unstable by flutter when friction is introduced.

\subsection{Numerical results for a disk brake}

A disk brake is considered with a finite element mesh using 17860 nodal points, $n=53580$ is the number of degrees of freedom. The pad is submitted to a uniform pressure on the upper face. Under the approximation $\mathcal{A}$, the steady sliding solution can be obtained within 3 iterations. This pressure is strictly positive on the whole contact surface, thus the assumption of effective contact is satisfied. The resolution of (133) is again done with a basis of functions composed of $m=200$ first vibration modes of the perfect system. For $f=0.4$, the unstable modes among the first 70 eigenmodes are modes $19,24,30,32,40,48,51,56,60,63,66,68$ of frequencies 5275,6483 , $8574,9113,11418,11951,12610,13721,14716,15302,15534,15746 \mathrm{~Hz}$ respectively. The real parts of the most unstable modes $32,48,51,68,63,66$ are shown in Figure 14. It is expected that, in the spirit of Hopf's bifurcation, after a flutter instability the dynamic response of the system will eventually become periodic with different phases of stick, slip and separation regimes as it can be observed in various examples of the literature, cf. Martins et al. (1999), Oden and Martins (1985), Vola et al. (1999). Brake squeal results as a consequence of this periodic regime. The frequencies of the periodic responses, if exist, are however not directly related to the flutter modes although they may remain close. From the analysis of the mechanism of flutter, our analysis leads already to some suggestions in order to impede such an instability. 


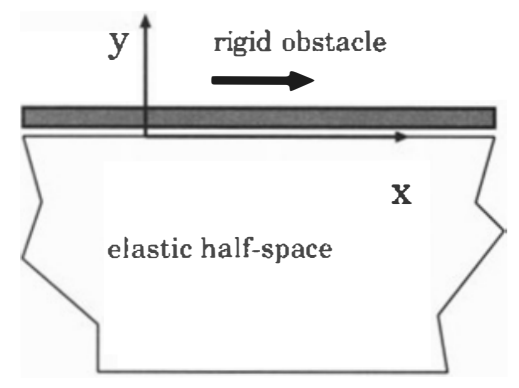

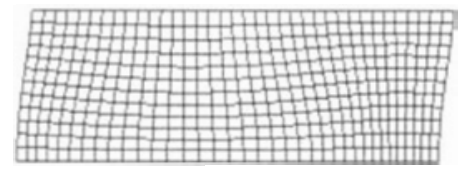

mode $2.13357 \mathrm{~Hz}$

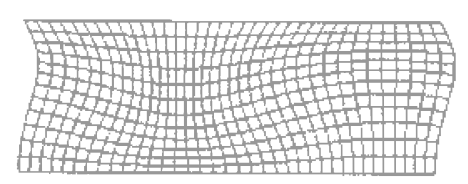

mode $5,1428.3 \mathrm{~Hz}$

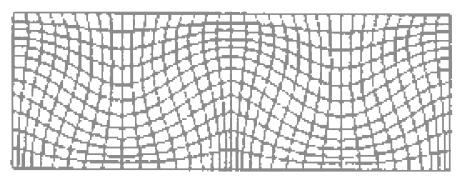

mode $8.16150 \mathrm{~Hz}$

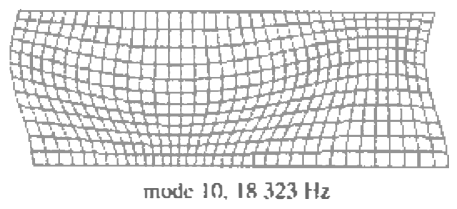

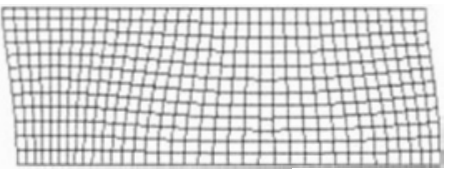

monde 3,13357 112

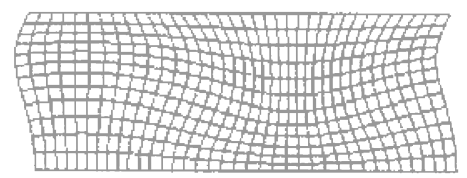

ituede 6, $1428.3 \mathrm{~Hz}$

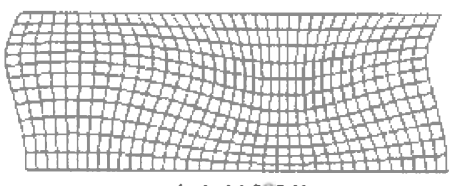

mode 6, $14283 \mathrm{~Hz}$.

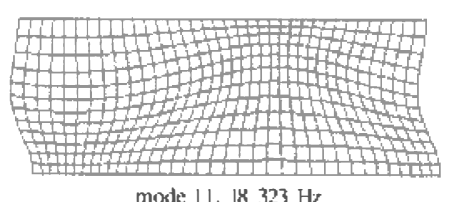

mode $11.18323 \mathrm{H} z$

Figure 13: Flutter instability of the steady sliding of a rigid plate on an infinite elstic layer: basis of vibration modes. 

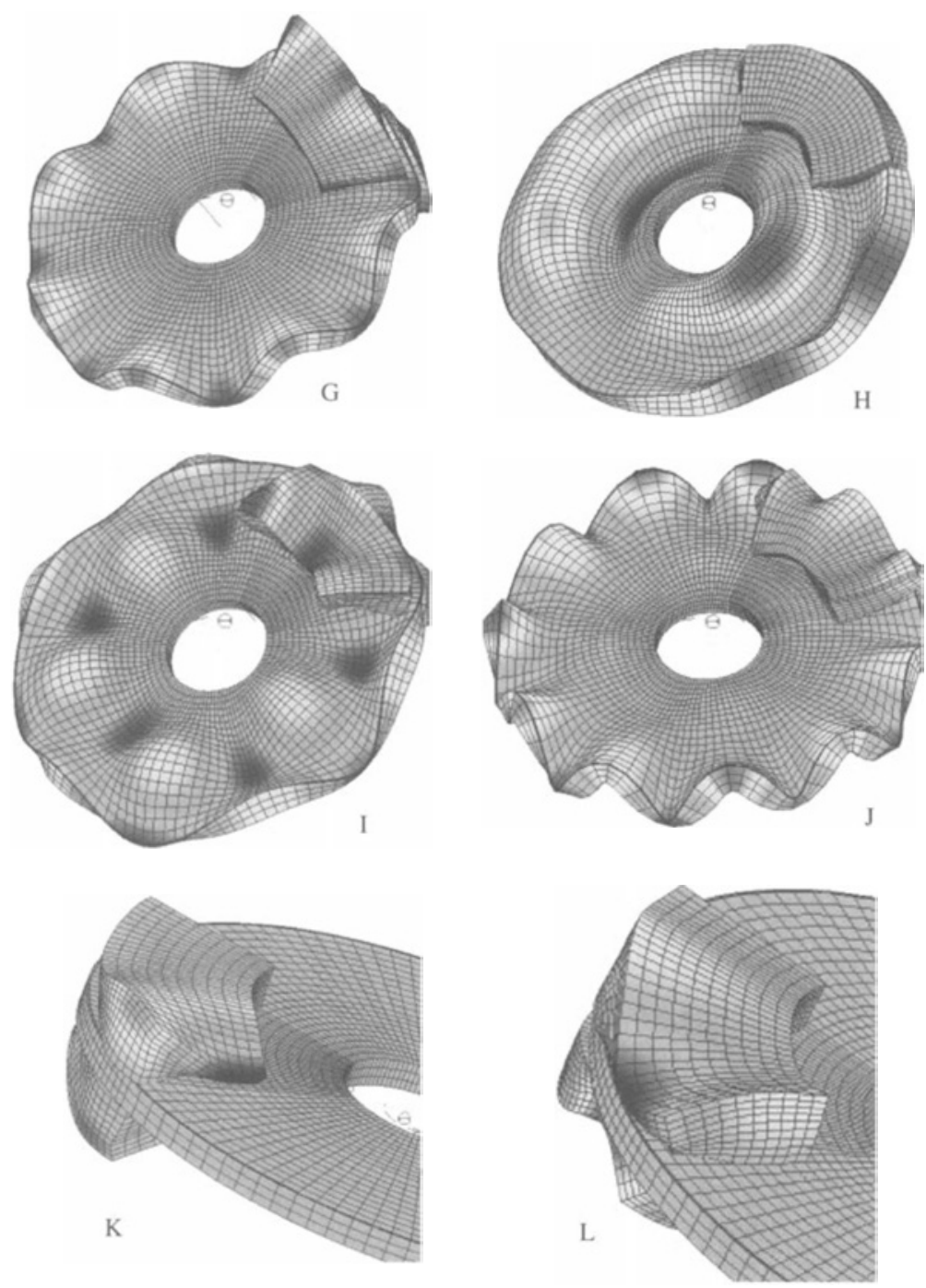

Figure 14: Flutter instability of the steady sliding solution of the pad-disk system: the real parts of some unstable modes are presented. 


\section{References}

Adams, G. (1995). Self-excited oscillations of two elastic half-spaces sliding with a constant coefficient of friction. J. Appl. Mech. 62:867-872.

Bazant, Z., and Cedolin, L. (1991). Stability of structures. Elastic, plastic, fracture and damage theories. Oxford: Oxford University Press.

Biot, M. (1965). Mechanics of incremental deformation. New York: Wiley.

Budiansky, B. (1974). Theory of buckling and post-buckling behaviour of elastic structures. In Advances in Applied Mechanics, volume 14. New York: Academic Press. 1-65.

Chambrette, P., and Jezequel, L. (1992). Stability of a beam rubbed against a rotating disk. Eur. J. Mech., A/Solids 11:107-138.

Chateau, X., and Nguyen, Q. (1991). Buckling of elastic structures in unilateral contact with or without friction. Eur. J. Mech., A/Solids 10:71-89.

Cho, H., and Barber, J. (1999). Stability of the three-dimensional Coulomb friction law. Phil. Trans. R. Soc. London 455:839-861.

Cochard, A., and Madariaga, R. (1995). Dynamic faulting under rate-independent friction. Pure \& Appl. Geophys. 142:419-445.

Cocu, M., Pratt, E., and Raous, M. (1996). Analysis of an incremental formulation for frictional contact problems. In Contact mechanics, Marseille, 1995. New York: Plenum Press.

Coddington, E., and Levinson, N. (1955). Theory of ordinary differential equations. New York: McGrawHill.

Cottle, R., Pang, J., and Stone, R. (1992). The linear complementarity problem. New York: Academic Press.

Durand, S. (1996). Dynamique des systèmes à liaisons unilatérales avec frottement sec. Thèse, Ecole Nationale des Ponts et Chaussées, Paris.

Girardot, D. (1997). Stabilité et bifurcation dynamiques des systèmes discrets. Thèse, Ecole Polytechnique, Paris.

Hale, J., and Kocak, H. (1991). Dynamics and bifurcation. New York: Springer-Verlag.

Hill, R. (1958). A general theory of uniqueness and stability in elastic/plastic solids. J. Mech. Phys. Solids 6:236-249.

Hlavacek, I., Haslinger, J., Necas, J., and Lovisek, J. (1988). Solution of variational inequalities in Mechanics. Berlin: Springer-Verlag.

Hutchinson, J. (1974). Plastic buckling. In Advances in Applied Mechanics, volume 14. New York: Academic Press. 67-114.

Iooss, G., and Joseph, D. (1981). Elementary stability and bifurcation theory. New York: Springer-Verlag. Isac, G. (1992). Complementary problems. New York: Springer-Verlag, Lecture Note in Mathematics.

Jean, M., and Moreau, J. (1987). Dynamics in the presence of unilateral contact and dry friction: a numerical approach. In Unilateral problems in structural analysis. Wien: CISM Course 304, Springer-Verlag. $1-50$.

Klarbring, A. (1986). A mathematical programming approach to three-dimensional contact problems with friction. Comp. Meth. Appl. Mech. Engng. 58:175-200.

Klarbring, A. (1990). Derivation and analysis of rate boundary value problems of frictional contact. Eur. J. Mech. A/Solids 9:53-85.

Klarbring, A. (1997). Contact, friction, discrete mechanical structures and mathematical programming. In Contact problems: theory, methods, applications. Wien: CISM Course, Springer-Verlag. 1-50.

Koiter, W. (1945). Over de stabiliteit van het elastisch evenwicht. Thesis, University of Delft. English translation AFFDL TR 70-25 (1970).

Marsden, J., and McCracken, M. (1976). The Hopf bifurcation and its applications. New York: SpringerVerlag. 
Martins, J., Guimaraes, J., and Faria, L. (1995). Dynamic surface solutions in linear elasticity and viscoelasticity with frictional boundary conditions. J. Vibration and Acoustics 117:445-451.

Martins, J., Barbarin, S., Raous, M., and Pinto da Costa, A . (1999). Dynamic stability of finite dimensional linear elastic system with unilateral contact and Coulomb's friction. Comp. Meth. Appl. Mech. Engng. 177:298-328.

Moirot, F., and Nguyen, Q. (2000a). Brake squeal: a problem of flutter instability of the steady sliding solution? Arch. Mech. 52:645-662.

Moirot, F., and Nguyen, Q. (2000b). An example of stick-slip waves. C. R. Acad. Sc. IIb, 328:663-669.

Moirot, F. (1998). Etude de la stabilité d'un équilibre en présence du frottement de Coulomb. Application au crissement des freins à disque. Thèse, Ecole Polytechnique, Paris.

Nguyen, Q. (1994). Bifurcation and stability in dissipative media (plasticity, friction, fracture). Appl. Mech. Rev. 47:1-31.

Nguyen, Q. (2000). Stability and Nonlinear Solid Mechanics. Chichester: Wiley.

Oancea, V., and Laursen, T. (1997). Stability analysis of state-dependent dynamic frictional sliding. Int. J. Nonlinear Mech. 32:837-853.

Oden, J., and Martins, J. (1985). Models and computational methods for dynamic friction phenomena. Comp. Meth. Appl. Mech. Engng. 52:527-634.

Oestreich, M., Hinrichs, N., and Popp, K. (1996). Bifurcation and stability analysis for a non-smooth friction oscillator. Arch. Appl. Mech. 66:301-314.

Popp, K., and Stelter, P. (1990). Stick-slip vibrations and chaos. Phil. Trans. R. Soc. Lond A, 332:89-105.

Renard, Y. (1998). Modélisation des instabilités liées au frottement sec des solides, aspects théoriques et numériques. Thèse de doctorat, Université de Grenoble.

Troger, H., and Steindl, A. (1991). Nonlinear stability and bifurcation theory. Wien: Springer-Verlag.

Vola, D., Raous, M., and Martins, J. (1999). Friction and instability of steady sliding squeal of a glass/rubber contact. Int. J. Num. Meth. Engng. 45:301-314.

Zharii, O. (1996). Frictional contact between the surface wave and a rigid strip. J. Appl. Mech. 63:15-20. 